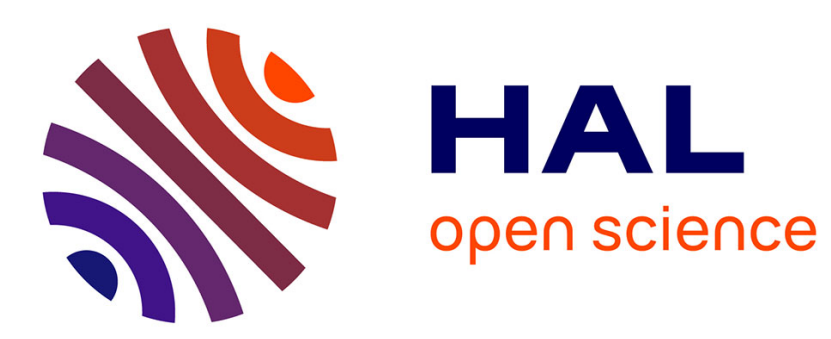

\title{
NECESSARY OPTIMALITY CONDITIONS FOR INFINITE DIMENSIONAL STATE CONSTRAINED CONTROL PROBLEMS
}

Hélène Frankowska, E M Marchini, M Mazzola

\section{> To cite this version:}

Hélène Frankowska, E M Marchini, M Mazzola. NECESSARY OPTIMALITY CONDITIONS FOR INFINITE DIMENSIONAL STATE CONSTRAINED CONTROL PROBLEMS. Journal of Differential Equations, 2018, 264, pp.7294-7327. hal-02126109

\section{HAL Id: hal-02126109 \\ https://hal.science/hal-02126109}

Submitted on 10 May 2019

HAL is a multi-disciplinary open access archive for the deposit and dissemination of scientific research documents, whether they are published or not. The documents may come from teaching and research institutions in France or abroad, or from public or private research centers.
L'archive ouverte pluridisciplinaire HAL, est destinée au dépôt et à la diffusion de documents scientifiques de niveau recherche, publiés ou non, émanant des établissements d'enseignement et de recherche français ou étrangers, des laboratoires publics ou privés. 


\title{
NECESSARY OPTIMALITY CONDITIONS FOR INFINITE DIMENSIONAL STATE CONSTRAINED CONTROL PROBLEMS
}

\author{
H. FRANKOWSKA, E.M. MARCHINI, AND M. MAZZOLA
}

\begin{abstract}
This paper is concerned with first order necessary optimality conditions for state constrained control problems in separable Banach spaces. Assuming inward pointing conditions on the constraint, we give a simple proof of Pontryagin maximum principle, relying on infinite dimensional neighbouring feasible trajectories theorems proved in [20]. Further, we provide sufficient conditions guaranteeing normality of the maximum principle. We work in the abstract semigroup setting, but nevertheless we apply our results to several concrete models involving controlled PDEs. Pointwise state constraints (as positivity of the solutions) are allowed.
\end{abstract}

\section{InTRODUCTION}

The maximum principle for optimal control problems can be considered as a milestone in the theory of control. Due to its importance, an extensive literature has been devoted to this subject, both in finite and in infinite dimensions. The main interest of the infinite dimensional setting is due to the fact that many physical models can be formulated in this framework, as for instance heat conduction, reaction-diffusion processes, properties of elastic materials, to mention only a few of them. To optimize a measure of best performance is indeed a natural need in concrete problems. For this reason optimal control governed by PDEs is a very active field of research, see e.g. the classical books, $[6,8,9,17,24,25,35]$, containing also rich bibliographies. In the literature two strategies can be found to deal with such an interesting topic: the abstract semigroup approach, and a direct one relying on PDEs methods. The advantage of the second approach is that many fine properties of the solutions, as regularity, can be used. In contrast, the first more general framework directly applies to a variety of models. Further, some of the techniques developed in finite dimensions can be adapted (modulo fine, and sometimes difficult, tuning) to this setting.

Among the two approaches we exploit here the first one. Nevertheless, we are convinced that some of the introduced here technical methods can be adapted also in the direct PDEs analysis. Previously, we have developed some tools in the abstract setting, see [19, 20], suitable to deal with state constrained problems, that are of crucial importance in applied sciences. In particular, we have proved some neighbouring feasible trajectory theorems allowing to estimate the distance between a given trajectory of an evolution system and its trajectories lying in the interior of the state constraint. This tool has been studied in depth in the finite dimensional setting, see e.g. $[12,31,32]$ to mention a few. To our knowledge

2010 Mathematics Subject Classification. 49K20, 49K27, 49K30, 35Q93.

Key words and phrases. semilinear differential inclusion, optimal control, state constraints, maximum principle, normality. 
in the infinite dimensional framework, neighbouring feasible trajectory theorems have been proved for the first time in [20]. In the present paper, using this effective tool, we provide a direct proof of the Pontryagin maximum principle for an optimal control state constrained problem, together with its normality. Further, we apply our results to some concrete models involving controlled PDEs with state constraints and study some examples with pointwise state constraints, such as positivity of solutions (important when dealing with populations dynamics) or lower and upper pointwise bounds (important in heat equations to avoid damage in the material during heating processes).

In an infinite dimensional separable Banach space $X$, we consider the solutions $x: I=$ $[0,1] \rightarrow X$ of the control system

$$
\dot{x}(t)=\mathbb{A} x(t)+f(t, x(t), u(t)), \quad \text { a.e. } t \in I,
$$

that satisfy an initial condition of the form

$$
x(0) \in Q_{0}
$$

and the state constraint

$$
x(t) \in K, \quad \forall t \in I .
$$

Here, $u$ is a measurable selection of a given measurable set valued map $U: I \rightsquigarrow Z$ with closed non-empty images, and $Z$ is a complete separable metric space modeling the control set. The densely defined unbounded linear operator $\mathbb{A}$ is the infinitesimal generator of a strongly continuous semigroup $S(t): X \rightarrow X$, the map $f: I \times X \times Z \rightarrow X$ is Fréchet differentiable with respect to the second variable $x, Q_{0}$ and $K$ are closed subsets of $X$. The trajectories of (1.1) are understood in the mild sense (see [29]). Notice that we allow nonsmooth constraints, that are important in the applications (industrial, medical, economical...). In this paper we analyze a constrained infinite dimensional Mayer problem: given a differentiable map $g: X \rightarrow \mathbb{R}$, consider the solutions of the problem

$$
\text { (1.4) minimize }\{g(x(1)): x \text { is a solution of }(1.1),(1.2),(1.3) \text { for some control } u(\cdot)\} \text {. }
$$

Recall that optimal control problems involving the integral cost can be reduced to (1.4) by adding an extra variable. Our main result is a direct proof of the following constrained Pontryagin Maximum Principle (PMP): given a locally optimal trajectory/control pair $(\bar{x}, \bar{u})$ for problem (1.4), there exist a multiplier $\lambda \in\{0,1\}$, a countably additive regular measure of bounded variation $\gamma$ such that the solution $z$ to the measure-driven adjoint variational equation

$$
\left\{\begin{array}{l}
\mathrm{d} z(t)=-\left(\mathbb{A}^{*}+\partial_{x} f(t, \bar{x}(t), \bar{u}(t))^{*}\right) z(t) \mathrm{d} t-\gamma(\mathrm{d} t), \quad t \in I \\
z(1)=\lambda \nabla g(\bar{x}(1))
\end{array}\right.
$$

satisfies the optimality condition

$$
\langle z(t), f(t, \bar{x}(t), \bar{u}(t))\rangle=\min _{u \in U(t)}\langle z(t), f(t, \bar{x}(t), u)\rangle, \quad \text { for a.e. } t \in I
$$

together with a transversality condition (4.4) at $t=0$, and $(\lambda, z(\cdot)) \neq(0,0)$. The solution of (1.5) is understood in the sense of [17] (see section 4 for the details).

The maximum principle in Banach spaces has been studied in the 60ies, see the papers $[14,15]$. Since then, many authors have contributed to extend it to the state constrained 
case, both in the abstract semigroup setting and in the PDEs framework. Due to the great literature on the subject we cannot provide an exhaustive list of contributions, among many others we mention $[2,5,7,10,11,23,26,28,30]$, the classical books quoted above, and the references contained therein. The novelty of our paper relies in the fully general state constrained evolutionary systems considered. For these problems we are able to provide a simple proof of the constrained (PMP) together with sufficient conditions implying the validity of the optimality condition in a qualified (normal) form as explained below.

To derive this result instead of using Ekeland's principle, as it was done in many papers dealing with necessary conditions, both in the abstract semigroup setting and in the direct approach to PDEs, see e.g. [17], we apply a direct variational approach based on our results from [20]. The main idea is to linearize the constrained control problem, using convexified variational differential inclusions and a convex linearization of the state constraints, to prove the generalized Fermat rule. Then, the duality arguments lead to necessary conditions for optimality. Moreover, exploiting inward pointing conditions, we guarantee that (PMP) holds in normal form, that is with $\lambda=1$ (see [21] for an overview of the existing results in finite dimension). Normality of the maximum principle plays a crucial role in necessary optimality conditions since it allows to deduce qualitative properties of the optimal trajectories, while in the abnormal case, the (PMP) does not depend on the cost function.

The paper is organized as follows: in section 2 we provide definitions and hypotheses in use; section 3 is devoted to some results dealing with the main assumption on the state constraint $K$, namely the inward pointing condition; sections 4 and 6 contain the proof of (PMP) without and with endpoint constraint respectively; section 5 discusses some concrete systems governed by controlled PDEs fitting our abstract model; in the last section 7 we prove some technical lemmas.

\section{Preliminaries}

In this section we list the notation and the main assumptions in use throughout the paper.

\subsection{Notation.}

- $B(x, r)$ denotes the closed ball of center $x \in X$ and radius $r>0 ; B$ is the closed unit ball in $X$ centered at $0 ; I=[0,1] ; \mu$ is the Lebesgue measure on the real line;

- $\overline{c o} K$ is the closed convex hull of a set $K \subset X$;

- given a Banach space $Y, \mathbb{L}(X, Y)$ denotes the Banach space of bounded linear operators from $X$ into $Y, \mathcal{C}(I, X)$ the space of continuous functions from $I$ to $X$, $L^{1}(I, X)$ the space of Bochner integrable functions from $I$ to $X$, and $L^{\infty}(I, X)$ the space of measurable essentially bounded functions from $I$ to $X, \mathcal{M}\left(I, X^{*}\right)$ the space of countably additive regular measures of bounded variation on $I$, isomorphic to the dual space of $\mathcal{C}(I, X)$, see [17];

$-\langle\cdot, \cdot\rangle$ stands for the duality pairing on $X^{*} \times X$; 
- given a set-valued map $\mathcal{F}: X \rightsquigarrow X, x \in X$ and $y \in \mathcal{F}(x)$, the derivative $d \mathcal{F}(x, y)$ : $X \rightsquigarrow X$ is defined by

$$
v \in d \mathcal{F}(x, y) w \quad \Leftrightarrow \quad \lim _{h \rightarrow 0+} \operatorname{dist}\left(v, \frac{\mathcal{F}(x+h w)-y}{h}\right)=0 ;
$$

- the distance from $x \in X$ to a nonempty subset $K \subset X$ is defined by

$$
\operatorname{dist}_{K}(x)=\inf _{k \in K}\|x-k\|_{X} ;
$$

- the contingent, Dubovitskij-Miljutin and Clarke tangent cones to a closed set $K$ at $x \in K$ are defined respectively by

$$
\begin{gathered}
T_{K}(x)=\left\{v \in X: \liminf _{h \rightarrow 0^{+}} \frac{\operatorname{dist}_{K}(x+h v)}{h}=0\right\}, \\
\left.\left.D M_{K}(x)=\{v \in X: \exists \varepsilon>0 \text { such that } x+] 0, \varepsilon\right](v+\varepsilon B) \subset K\right\}, \\
C_{K}(x)=\left\{v \in X: \lim _{h \rightarrow 0^{+}, x^{\prime} \rightarrow K} \frac{\operatorname{dist}_{K}\left(x^{\prime}+h v\right)}{h}=0\right\} ;
\end{gathered}
$$

the Clarke normal cone $N_{K}(x) \subseteq X^{*}$ is the negative polar cone of $C_{K}(x)$;

- the oriented (or signed) distance from $x \in X$ to $K$ is defined as

$$
d_{K}(x)= \begin{cases}\inf _{k \in K}\|x-k\|_{X} & \text { if } x \notin K \\ -\inf _{k \in(X \backslash K)}\|x-k\|_{X} & \text { otherwise }\end{cases}
$$

- $\partial d_{K}(x)$ denotes the Clarke generalized gradient of $d_{K}$ at $x \in X$, while the generalized directional derivative of $d_{K}$ at $x \in X$ in the direction $v \in X$ is defined by

$$
d_{K}^{0}(x ; v)=\limsup _{h \rightarrow 0^{+}, x^{\prime} \rightarrow x} \frac{d_{K}\left(x^{\prime}+h v\right)-d_{K}\left(x^{\prime}\right)}{h} ;
$$

- for every $x \in X$, consider the support function $\sigma(x ; \cdot): X^{*} \rightarrow \mathbb{R}$ of $\partial d_{K}(x)$ defined by

$$
\sigma(x ; y)=\sup _{\xi \in \partial d_{K}(x)}\langle\xi, y\rangle, \quad \forall y \in X
$$

- for any $\eta>0$ define the set

$$
\partial^{\eta} K=\{x \in K+\eta B: S(\tau) x \in \partial K+\eta B \text { for some } \tau \in[0, \eta]\},
$$

where $S(\cdot)$ is the strongly continuous semigroup referred to in the introduction, and for every $x \in X$, the set

$$
\mathcal{A}^{\eta}(x)=\{(\tau, z) \in[0, \eta] \times X: S(\tau) x \in \partial K+\eta B, z \in B(S(\tau) x, \eta)\},
$$

and the function $\Sigma_{\eta}(x ; \cdot): X \rightarrow[-\infty,+\infty)$ that associates with any $v \in X$ the value

$$
\Sigma_{\eta}(x ; v)=\sup _{(\tau, z) \in \mathcal{A}^{\eta}(x)} \sigma(z ; S(\tau) v),
$$

with the convention that the supremum over the empty set is equal to $-\infty$. 
Definition 2.1. Let $x_{0} \in X$. A function $x \in \mathcal{C}(I, X)$ is a (mild) solution of (1.1) with initial datum $x(0)=x_{0}$ if it satisfies

$$
x(t)=S(t) x_{0}+\int_{0}^{t} S(t-s) f(s, x(s), u(s)) \mathrm{d} s, \quad \text { for any } t \in I,
$$

for some measurable selection $u$ of the set valued map $t \rightsquigarrow U(t)$. If in addition $x$ satisfies (1.2)-(1.3), we say that $(x, u)$ is an admissible pair for problem (1.4).

Definition 2.2. Given a set-valued map with closed non-empty images $F: I \times X \rightsquigarrow X$, and $x_{0} \in X$, a function $x \in \mathcal{C}(I, X)$ is a (mild) solution of

$$
\dot{x}(t) \in \mathbb{A} x(t)+F(t, x(t)),
$$

with initial datum $x(0)=x_{0}$, if there exists a function $f^{x} \in L^{1}\left(\left[t_{0}, 1\right], X\right)$ such that

$$
f^{x}(t) \in F(t, x(t)), \quad \text { for a.e. } t \in I
$$

and

$$
x(t)=S(t) x_{0}+\int_{0}^{t} S(t-s) f^{x}(s) \mathrm{d} s, \quad \text { for any } t \in I .
$$

Notice that, since $S(\cdot)$ is a strongly continuous semigroup, there exists $M_{S}>0$ such that

$$
\|S(t)\|_{\mathbb{L}(X, X)} \leq M_{S}, \quad \text { for any } t \in I .
$$

2.2. Assumptions. For every $(t, x) \in I \times X$, set $F(t, x)=f(t, x, U(t))$. The following conditions $(\mathrm{H})$ are imposed in the main results:

(i) $Q_{0}$ and $K$ are nonempty closed subsets of $X ; K$ is positively invariant with respect to the semigroup $S(\cdot)$, i.e.

$$
S(t) K \subset K, \quad \forall t \geq 0
$$

$$
U: I \rightsquigarrow Z \text { is measurable with nonempty closed values; }
$$

$f$ is measurable in $t$, Fréchet differentiable in $x$ and continuous in $u$;

(iii) for any $R>0$, there exists $k_{R} \in L^{1}\left(I, \mathbb{R}^{+}\right)$such that, for a.e. $t \in I$ and any $u \in Z$,

$$
f(t, \cdot, u) \text { is } k_{R}(t)-\text { Lipschitz on } B(0, R) \text {; }
$$

(iv) for a.e. $t \in I$ and all $x \in X, F(t, x)$ is closed and there exists $\phi \in L^{1}\left(I, \mathbb{R}^{+}\right)$such that, for a.e. $t \in I$ and any $x \in X$,

$$
F(t, x) \subset \phi(t)\left(1+\|x\|_{X}\right) B .
$$

(v) main inward pointing condition (IPC):

$$
\forall R>0, \exists \eta, \rho, M>0, \exists J \subset I \text { such that } \mu(J)=1 \text { and if } \Sigma_{\eta}(x ; v) \geq 0
$$

for some $t \in J, x \in R B \cap \partial^{\eta} K, v \in \overline{\mathrm{co}} F(t, x)$, then $\exists \bar{v} \in \overline{\mathrm{co}} F(t, x) \cap B(v, M)$ satisfying

$$
\max \left\{\Sigma_{\eta}(x ; \bar{v}-v) ; \Sigma_{\eta}(x ; \bar{v})\right\} \leq-\rho .
$$

$g$ is Fréchet differentiable. 
Sometimes, simplified versions of the inward pointing condition can be used. For example, under convexity and compactness assumptions (see Remarks 4.4 and 4.5 below), the technical condition (2.8) can be replaced by

$$
\begin{gathered}
\forall R>0, \exists \rho>0 \text { such that } \forall \bar{x} \in \partial K \cap R B, \\
\text { if } \sigma(\bar{x} ; v) \geq 0 \text { for some } t \in I, v \in F(t, \bar{x}) \text {, then } \inf _{\bar{v} \in F(t, \bar{x})} \sigma(\bar{x} ; \bar{v}-v) \leq-\rho .
\end{gathered}
$$

or, in the case when $K$ has a smooth boundary, by the classical inward pointing condition proposed by Soner in [33]:

$$
\forall R>0, \exists \rho>0 \text { such that } \inf _{\bar{v} \in F(t, \bar{x})}\left\langle\nabla d_{K}(\bar{x}), \bar{v}\right\rangle \leq-\rho, \forall(t, \bar{x}) \in I \times(\partial K \cap R B) .
$$

Proposition 2.3. [20, Lemma 5.1] Let F satisfy (2.7) with $\phi \in L^{\infty}\left(I, \mathbb{R}^{+}\right)$. Then, the simplified condition

$$
\begin{gathered}
\forall R>0, \exists \eta, \rho>0, \exists J \subset I \text { such that } \mu(J)=1 \text { and if } \Sigma_{\eta}(x ; v) \geq 0 \\
\text { for some } t \in J, x \in R B \cap \partial^{\eta} K, v \in \overline{\mathrm{co}} F(t, x), \text { then } \exists \bar{v} \in \overline{\mathrm{co}} F(t, x) \text { satisfying } \\
\Sigma_{\eta}(x ; \bar{v}-v) \leq-\rho,
\end{gathered}
$$

implies (2.8).

\section{Inward pointing Condition and Clarke tangent Cone}

In this section we point out some consequences of the inward pointing condition. In particular we prove that under the assumption (2.8), the interior of the Clarke tangent cone is nonempty at every boundary point of $K$. This property will be crucial in the study of necessary optimality conditions.

Since the inward pointing condition is formulated by means of the Clarke generalized gradient of $d_{K}$, for every $x \in K$ we introduce the cone

$$
D_{K}(x)= \begin{cases}\{v \in X: \sigma(x ; v)<0\} & \text { if } x \in \partial K \\ X & \text { otherwise }\end{cases}
$$

The link between $D_{K}(x)$ and the Clarke tangent cone $C_{K}(x)$ follows from the next proposition.

Proposition 3.1. For every $x \in K$ we have

$$
D_{K}(x) \subseteq \operatorname{Int} C_{K}(x)
$$

and

$$
D_{K}(x) \subseteq D M_{K}(x) .
$$

Proof. Let $x \in \partial K$, otherwise the claim is trivial, and $v \in X$ satisfy $\sigma(x ; v) \leq 0$. We first claim that $v \in C_{K}(x)$. Given $h_{i} \rightarrow 0+$ and $x_{i} \rightarrow x$ in $K$, we have to prove that for every $i \in \mathbb{N}$ there exists $v_{i} \in X$ such that $x_{i}+h_{i} v_{i} \in K$ and $v_{i} \rightarrow v$ as $i \rightarrow+\infty$. Set

$$
J=\left\{i \in \mathbb{N}: x_{i}+h_{i} v \notin K\right\} .
$$


If $J$ is finite, it is sufficient to consider $v_{i}=v$ for all $i \in \mathbb{N}$ to conclude. Otherwise, we have

$$
0 \leq \limsup _{i \rightarrow \infty, i \in J} \frac{\operatorname{dist}_{K}\left(x_{i}+h_{i} v\right)-\operatorname{dist}_{K}\left(x_{i}\right)}{h_{i}} \leq \limsup _{i \rightarrow \infty, i \in J} \frac{d_{K}\left(x_{i}+h_{i} v\right)-d_{K}\left(x_{i}\right)}{h_{i}} \leq d_{K}^{0}(x ; v) .
$$

Recalling that

$$
d_{K}^{0}(x ; v)=\sup _{\xi \in \partial d_{K}(x)}\langle\xi, v\rangle,
$$

and that $\sigma(x ; v) \leq 0$, we deduce from (3.3) that

$$
\lim _{i \rightarrow \infty, i \in J} \frac{\operatorname{dist}_{K}\left(x_{i}+h_{i} v\right)}{h_{i}}=0 .
$$

Then, for every $i \in J$ there exists $w_{i} \in K$ such that $\frac{x_{i}+h_{i} v-w_{i}}{h_{i}} \rightarrow 0$. For any $i \notin J$, set $v_{i}=\frac{w_{i}-x_{i}}{h_{i}}$ for any $i \in \mathbb{N}$, we obtain that $v_{i} \rightarrow v$ and $x_{i}+h_{i} v_{i}=w_{i} \in K$, ending the proof of the claim.

Now, consider any $v \in D_{K}(x)$ and let $\varepsilon>0$ be such that $\sigma(x ; v)=-\varepsilon<0$. Hence

$$
\langle\xi, v+\varepsilon z\rangle \leq\langle\xi, v\rangle+\varepsilon \leq 0, \quad \forall \xi \in \partial d_{K}(x), \forall z \in B .
$$

Recalling the claim, we conclude that $v \in \operatorname{Int} C_{K}(x)$.

In order to prove (3.2), let us suppose by contradiction that $v \in D_{K}(x) \backslash D M_{K}(x)$. Since $D M_{K}(x)$ is the complement to the contingent cone to the complement of $K$ at $x$ (see [4]), there exist sequences $h_{i} \rightarrow 0+$ and $v_{i} \rightarrow v$ satisfying $x+h_{i} v_{i} \notin K$ for every $i \in \mathbb{N}$. By the mean value theorem (see [13]), for any $i \in \mathbb{N}$ there exists $\xi_{i} \in \partial d_{K}\left(z_{i}\right)$, for some $z_{i}$ belonging to the segment $\left[x, x+h_{i} v_{i}\right]$, satisfying

$$
d_{K}\left(x+h_{i} v_{i}\right)=d_{K}(x)+\left\langle\xi_{i}, h_{i} v_{i}\right\rangle .
$$

Consequently,

$$
0<\left\langle\xi_{i}, v_{i}\right\rangle=\left\langle\xi_{i}, v_{i}-v\right\rangle+\left\langle\xi_{i}, v\right\rangle \leq\left\|v_{i}-v\right\|_{X}+\left\langle\xi_{i}, v\right\rangle, \quad \forall i \in \mathbb{N} .
$$

We can assume (up to a subsequence) that $\xi_{i} \stackrel{*}{\rightarrow} \xi$ weakly-star in $X^{*}$. Recalling that $z_{i} \rightarrow x$ and that the Clarke generalized gradient is weak*-upper semicontinuous (see [13]), we obtain $\xi \in \partial d_{K}(x)$. Passing to the limit in (3.4) as $i \rightarrow \infty$, we obtain a contradiction with the assumption $v \in D_{K}(x)$.

Proposition 3.2. Assume (2.8). Then $D_{K}(x) \neq \emptyset$ for every $x \in K$.

Proof. Consider $x \in \partial K$, otherwise the statement is trivial. Setting $R=\|x\|_{X}$, let $\eta>0$ be as in (2.8). We have to prove the existence of $\bar{v} \in X$ such that $\sigma(x ; \bar{v})<0$. Suppose by contradiction that $\sigma(x ; v) \geq 0$, for every $v \in X$. Then we have

$$
\Sigma_{\eta}(x, v)=\sup _{(\tau, z) \in \mathcal{A}^{\eta}(x)} \sigma(z ; S(\tau) v) \geq \sigma(x ; v) \geq 0, \quad \forall v \in X .
$$

By (2.8), there exists $\bar{v} \in X$ such that $\Sigma_{\eta}(x, \bar{v})<0$. In particular, we obtain $\sigma(x ; \bar{v})<0$, reaching a contradiction. 
Proposition 3.3. Assume (H) (i)-(v) and let $(x, u)$ be an admissible pair for (1.1), (1.3). For any $t \in I$, set

$$
\mathbb{B}(t)=\partial_{x} f(t, x(t), u(t)) \quad f^{x}(t)=f(t, x(t), u(t)) \quad \text { and } \quad \mathcal{T}(t)=T_{\overline{\mathrm{co}} f(t, x(t), U(t))}\left(f^{x}(t)\right)
$$

Then, for every $w_{0} \in D_{K}(x(0))$, there exists a solution of

$$
\left\{\begin{array}{l}
\dot{w}(t) \in \mathbb{A} w(t)+\mathbb{B}(t) w(t)+\mathcal{T}(t) \quad \text { a.e. } t \in I, \\
w(0)=w_{0},
\end{array}\right.
$$

that belongs to the interior of the set

$$
\left\{w \in \mathcal{C}(I, X): w(t) \in C_{K}(x(t)), \forall t \in I\right\} .
$$

The proof of Proposition 3.3 is postponed to section 7.

\section{A CONSTRAined MAXIMUM PRINCIPLE}

We say that an admissible pair $(\bar{x}, \bar{u})$ is locally optimal for problem (1.4) if there exists $\delta>0$ such that, for any admissible $(x, u)$ with $\|x-\bar{x}\|_{L^{\infty}(I, X)}<\delta$, we have $g(x(1)) \geq g(\bar{x}(1))$. Given a locally optimal pair $(\bar{x}, \bar{u})$ for problem $(1.4)$, set, for any $t \in I, f^{\bar{x}}(t)=f(t, \bar{x}(t), \bar{u}(t))$ and $\mathcal{T}(t)=T_{\overline{\mathrm{co}} f(t, \bar{x}(t), U(t))}\left(f^{\bar{x}}(t)\right)$. Consider the following variational inclusion

$$
\left\{\begin{array}{l}
\dot{w}(t) \in \mathbb{A} w(t)+\partial_{x} f(t, \bar{x}(t), \bar{u}(t)) w(t)+\mathcal{T}(t) \quad \text { a.e. } t \in I, \\
w(0) \in C_{0},
\end{array}\right.
$$

where $C_{0}$ is a convex cone contained in $T_{K \cap Q_{0}}(\bar{x}(0))$. Define $F(t, x)=f(t, x, U(t))$ for every $(t, x) \in I \times X$. From assumption (2.6), it follows that $F$ is locally Lipschitz continuous with respect to the variable $x$. Then, as in [18], for a.e. $t \in I$ and $w \in X$, we have

$$
\partial_{x} f(t, \bar{x}(t), \bar{u}(t)) w \in d F\left(t, \bar{x}(t), f^{\bar{x}}(t)\right) w
$$

and

$$
\partial_{x} f(t, \bar{x}(t), \bar{u}(t)) w+\mathcal{T}(t) \subseteq d \overline{\mathrm{co}} F\left(t, \bar{x}(t), f^{\bar{x}}(t)\right) w,
$$

where $d F(t, \alpha, \beta)$ (respectively $d \overline{\mathrm{co}} F(t, \alpha, \beta)$ ) denotes the derivative of $F(t, \cdot)$ (respectively $\overline{\mathrm{co}} F(t, \cdot))$ at $(\alpha, \beta)$.

Proposition 4.1. Assume that $(\bar{x}, \bar{u})$ is locally optimal and that (H) hold true. Then every solution of

$$
\left\{\begin{array}{l}
\dot{w}(t) \in \mathbb{A} w(t)+d \overline{\operatorname{co}} F\left(t, \bar{x}(t), f^{\bar{x}}(t)\right) w(t) \quad \text { a.e. } t \in I \\
w(t) \in C_{K}(\bar{x}(t)) \quad \forall t \in I \\
w(0) \in T_{K \cap Q_{0}}(\bar{x}(0))
\end{array}\right.
$$

satisfies

$$
\langle\nabla g(\bar{x}(1)), w(1)\rangle \geq 0
$$


Proof. To prove the claim we apply a consequence of neighboring feasible trajectories theorems and relaxation results from [20]. Let $w$ be a solution to (4.2). Since $w(0) \in$ $T_{K \cap Q_{0}}(\bar{x}(0))$, there exist $h_{i} \rightarrow 0+$ and $w_{i} \rightarrow w(0)$ such that $\bar{x}(0)+h_{i} w_{i} \in K \cap Q_{0}$, for every $i \in \mathbb{N}$. Moreover, the following condition holds,

$$
\lim _{h \rightarrow 0^{+}} \frac{1}{h} \max _{t \in I} \operatorname{dist}_{K}(\bar{x}(t)+h w(t))=0 .
$$

Indeed assume, by contradiction, that

$$
\limsup _{h \rightarrow 0+} \max _{t \in I} \frac{\operatorname{dist}_{K}(\bar{x}(t)+h w(t))}{h}>0 .
$$

Then there exists $\varepsilon>0$ such that, for all $i \in \mathbb{N}$, we can find $h_{i} \leq 1 / i$ and $t_{i} \in[0,1]$ satisfying

$$
\frac{\operatorname{dist}_{K}\left(\bar{x}\left(t_{i}\right)+h_{i} w\left(t_{i}\right)\right)}{h_{i}} \geq \varepsilon
$$

Taking a subsequence and using the same notation, we may assume $t_{i} \rightarrow \bar{t} \in I$, implying

$$
\bar{x}\left(t_{i}\right) \rightarrow \bar{x}(\bar{t}), \quad w\left(t_{i}\right) \rightarrow w(\bar{t}) .
$$

Since $w(\bar{t}) \in C_{K}(\bar{x}(\bar{t}))$,

$$
\lim _{x^{\prime} \rightarrow_{K} \bar{x}(\bar{t}), h \rightarrow 0+, w^{\prime} \rightarrow w(\bar{t})} \frac{\operatorname{dist}_{K}\left(x^{\prime}+h w^{\prime}\right)}{h}=0 .
$$

in contradiction with (4.3).

Hence, the assumptions of [20, Theorem 7.1] are satisfied (replacing $F$ with $\overline{\mathrm{co}} F$ ), implying for any $i \in \mathbb{N}$ the existence of a solution $x_{i}$ to

$$
\left\{\begin{array}{l}
\dot{x}(t) \in \mathbb{A} x(t)+\overline{\mathrm{co}} F(t, x(t)) \quad \text { a.e. } t \in I, \\
x(t) \in K \quad \forall t \in I, \\
x(0)=\bar{x}(0)+h_{i} w_{i},
\end{array}\right.
$$

such that

$$
\frac{x_{i}-\bar{x}}{h_{i}} \rightarrow w \text { in } \mathcal{C}(I, X) .
$$

Applying a relaxation theorem [20, Theorem 4.5], for any $i \in \mathbb{N}$, we can find a solution $\bar{x}_{i}$ to $(1.1),(1.3)$ satisfying $\bar{x}_{i}(0)=x_{i}(0)$ and

$$
\left\|\bar{x}_{i}-x_{i}\right\|_{\mathcal{C}(I, X)}=o\left(h_{i}\right) .
$$

Consequently,

$$
\frac{\bar{x}_{i}-\bar{x}}{h_{i}} \rightarrow w \quad \text { in } \mathcal{C}(I, X) .
$$

Since $\bar{x}$ is locally optimal for the problem (1.4), for $i$ sufficiently large we have that

$$
g\left(\bar{x}_{i}(1)\right) \geq g(\bar{x}(1)),
$$

and therefore

$$
\langle\nabla g(\bar{x}(1)), w(1)\rangle=\lim _{i \rightarrow+\infty} \frac{g\left(\bar{x}_{i}(1)\right)-g(\bar{x}(1))}{h_{i}} \geq 0
$$


Below we shall use the notations of $[16,17]$.

Theorem 4.2. Assume (H). Let $(\bar{x}, \bar{u})$ be a locally optimal pair for problem (1.4), $C_{0}$ be any convex cone contained in $T_{K \cap Q_{0}}(\bar{x}(0))$ and $\mathcal{S}$ be the solution operator associated with

$$
\dot{w}(t)=\mathbb{A} w(t)+\partial_{x} f(t, \bar{x}(t), \bar{u}(t)) w(t) .
$$

Then, there exist $\lambda \in\{0,1\}$ and a measure

$$
\gamma \in\left(\left\{w \in \mathcal{C}(I, X): w(t) \in C_{K}(\bar{x}(t)), \forall t \in I\right\}\right)^{-}
$$

not vanishing simultaneously, such that the function $z: I \rightarrow X^{*}$ defined by

$$
z(s)=\mathcal{S}(1, s)^{*} \lambda \nabla g(\bar{x}(1))+\int_{s}^{1} \mathcal{S}(t, s)^{*} \gamma(\mathrm{d} t)
$$

satisfies the minimum principle (1.6) and the transversality condition

$$
-z(0) \in C_{0}^{-} \text {. }
$$

Furthermore, if

$$
D_{K}(\bar{x}(0)) \cap C_{0} \neq \emptyset,
$$

then the optimality condition holds also in the normal form: $\lambda=1$.

Remark 4.3. (i) If $X$ is reflexive, then $\mathbb{A}^{*}$ generates the strongly continuous semigroup $S^{*}(t)=S(t)^{*}$. In this case, $z$ is the mild solution of the adjoint equation (1.5), as in $[16,17]$.

(ii) If $C_{0}$ is the Clarke cone $C_{K \cap Q_{0}}(\bar{x}(0))$, the transversality condition (4.4) becomes

$$
-z(0) \in N_{K \cap Q_{0}}(\bar{x}(0)) \text {. }
$$

Proof of Theorem 4.2. Let

$$
\mathcal{D}=\left\{w \in \mathcal{C}(I, X): w(t) \in C_{K}(\bar{x}(t)), \forall t \in I\right\}
$$

and

$$
\mathcal{E}=\{w \in \mathcal{C}(I, X): w \text { is a mild solution to (4.1) }\} .
$$

Observe that $\mathcal{D}$ and $\mathcal{E}$ are nonempty convex cones. Furthermore, by Propositions 3.2 and 3.3, Int $\mathcal{D} \neq \emptyset$.

We have two different situations:

Case 1, abnormal PMP: Int $\mathcal{D} \cap \mathcal{E}=\emptyset$. Then, by a separation theorem, there exists $\beta \in(\mathcal{C}(I, X))^{*}, \beta \neq 0$, such that

$$
\inf _{d \in \mathcal{D}}\langle\beta, d\rangle \geq \sup _{e \in \mathcal{E}}\langle\beta, e\rangle
$$

Since $\mathcal{D}$ and $\mathcal{E}$ are cones,

$$
0=\inf _{d \in \mathcal{D}}\langle\beta, d\rangle=\sup _{e \in \mathcal{E}}\langle\beta, e\rangle
$$

and therefore

$$
\beta \in \mathcal{E}^{-} \quad \text { and } \quad-\beta \in \mathcal{D}^{-} .
$$


Case 2, normal PMP: Int $\mathcal{D} \cap \mathcal{E} \neq \emptyset$. Consider the linear operator $\Gamma: \mathcal{C}(I, X) \rightarrow X$ defined by $\Gamma(w)=w(1)$. Using a density argument, we deduce by Proposition 4.1 that

$$
0 \leq\langle\nabla g(\bar{x}(1)), w(1)\rangle=\langle\nabla g(\bar{x}(1)), \Gamma w\rangle=\left\langle\Gamma^{*} \nabla g(\bar{x}(1)), w\right\rangle,
$$

for any $w \in \mathcal{D} \cap \mathcal{E}$. Hence

$$
-\Gamma^{*} \nabla g(\bar{x}(1)) \in(\mathcal{D} \cap \mathcal{E})^{-} .
$$

Since $\mathcal{D}, \mathcal{E}$ are convex cones and $\operatorname{Int} \mathcal{D} \cap \mathcal{E} \neq \emptyset$, it is well known that

$$
(\mathcal{D} \cap \mathcal{E})^{-}=\mathcal{D}^{-}+\mathcal{E}^{-}
$$

(see e.g. [22] for a short proof of this fact), implying the existence of $\beta_{1} \in \mathcal{D}^{-}$and $\beta_{2} \in \mathcal{E}^{-}$ such that

$$
-\Gamma^{*} \nabla g(\bar{x}(1))=\beta_{1}+\beta_{2} .
$$

We can put together the two cases, by stating that there exist $\beta_{1} \in \mathcal{D}^{-}, \beta_{2} \in \mathcal{E}^{-}$and $\lambda \in\{0,1\}$ such that

$$
-\lambda \Gamma^{*} \nabla g(\bar{x}(1))=\beta_{1}+\beta_{2} \quad \text { and } \quad\left(\lambda, \beta_{1}\right) \neq(0,0)
$$

(in Case 1 we set $\beta_{1}=-\beta, \beta_{2}=\beta$ and $\lambda=0$ ). Observe that assumption (4.5) ensures that Int $\mathcal{D} \cap \mathcal{E} \neq \emptyset$, see Proposition 3.3. In this case, we can consider $\lambda=1$.

Representation: Let us consider any mild solution $w$ of (4.1). Then,

$$
w(t)=\mathcal{S}(t, 0) w_{0}+\int_{0}^{t} \mathcal{S}(t, s) v(s) \mathrm{d} s, \quad \text { for any } t \in I,
$$

where $w_{0}=w(0)$ and $v(s) \in \mathcal{T}(s)$, for a.e. $s \in I$, is integrable. Then, by taking $\lambda=0$ in Case 1 and $\lambda=1$ in Case 2, and applying the characterization of $\beta_{1} \in(C(I, X))^{*}$ from [16], we obtain for a measure $\gamma \in \mathcal{D}^{-} \subset \mathcal{M}\left(I, X^{*}\right)$,

$$
\begin{aligned}
0 \geq & \langle-\lambda \nabla g(\bar{x}(1)), w(1)\rangle-\left\langle\beta_{1}, w\right\rangle=-\left\langle\lambda \nabla g(\bar{x}(1)), \mathcal{S}(1,0) w_{0}+\int_{0}^{1} \mathcal{S}(1, s) v(s) \mathrm{d} s\right\rangle-\left\langle\beta_{1}, w\right\rangle \\
= & -\left\langle\mathcal{S}(1,0)^{*} \lambda \nabla g(\bar{x}(1)), w_{0}\right\rangle-\int_{0}^{1}\left\langle\mathcal{S}(1, s)^{*} \lambda \nabla g(\bar{x}(1)), v(s) \mathrm{d} s\right\rangle-\int_{0}^{1}\langle w(t), \gamma(\mathrm{d} t)\rangle \\
= & -\left\langle\mathcal{S}(1,0)^{*} \lambda \nabla g(\bar{x}(1)), w_{0}\right\rangle-\int_{0}^{1}\left\langle\mathcal{S}(1, s)^{*} \lambda \nabla g(\bar{x}(1)), v(s) \mathrm{d} s\right\rangle \\
& -\int_{0}^{1}\left\langle\mathcal{S}(t, 0) w_{0}+\int_{0}^{t} \mathcal{S}(t, s) v(s) \mathrm{d} s, \gamma(\mathrm{d} t)\right\rangle \\
= & -\left\langle\mathcal{S}(1,0)^{*} \lambda \nabla g(\bar{x}(1)), w_{0}\right\rangle-\int_{0}^{1}\left\langle\mathcal{S}(1, s)^{*} \lambda \nabla g(\bar{x}(1)), v(s) \mathrm{d} s\right\rangle \\
& -\left\langle\int_{0}^{1} \mathcal{S}(t, 0)^{*} \gamma(\mathrm{d} t), w_{0}\right\rangle-\int_{0}^{1}\left\langle\int_{s}^{1} \mathcal{S}(t, s)^{*} \gamma(\mathrm{d} t), v(s)\right\rangle \mathrm{d} s .
\end{aligned}
$$

In the last equality we applied [16, Lemma 4.1]. Now, let us define $z: I \rightarrow X^{*}$ by

$$
z(s)=\mathcal{S}(1, s)^{*} \lambda \nabla g(\bar{x}(1))+\int_{s}^{1} \mathcal{S}(t, s)^{*} \gamma(\mathrm{d} t) .
$$


According to [17, Theorem 10.2.15], $z$ is $X$-weakly measurable, meaning that

$$
t \mapsto\langle z(t), v\rangle \text { is measurable for any } v \in X .
$$

Plugging the expression of $z$ in the inequalities above we obtain

$$
\left\langle\mathcal{S}(1,0)^{*} \lambda \nabla g(\bar{x}(1))+\int_{0}^{1} \mathcal{S}(t, 0)^{*} \gamma(\mathrm{d} t), w_{0}\right\rangle+\int_{0}^{1}\langle z(s), v(s) \mathrm{d} s\rangle \geq 0 .
$$

Taking $v \equiv 0$ in (4.7) yields

$$
\left\langle\mathcal{S}(1,0)^{*} \lambda \nabla g(\bar{x}(1))+\int_{0}^{1} \mathcal{S}(t, 0)^{*} \gamma(\mathrm{d} t), w_{0}\right\rangle=\left\langle z(0), w_{0}\right\rangle \geq 0 .
$$

Since this holds true for every $w_{0} \in C_{0}$, we obtain the transversality condition

$$
-z(0) \in C_{0}^{-} .
$$

It remains to verify that $z$ satisfies the pointwise minimum principle (1.6). Set $w_{0}=0$ in the integral inequality (4.7). We have

$$
\int_{0}^{1}\langle z(t), v(t)\rangle \mathrm{d} t \geq 0
$$

for any integrable selection $v$ of the set valued map $I \ni t \rightsquigarrow T_{\overline{\mathrm{co}} f(t, \bar{x}(t), U(t))}\left(f^{\bar{x}}(t)\right)$. For every $i \in \mathbb{N}$, define the set-valued maps $V_{i}, W_{i}$ and $\tilde{W}_{i}$ from $I$ to subsets of $X$ by

$$
V_{i}(t)=\left\{v \in X:\langle z(t), v\rangle \leq-\frac{1}{i}\right\}, \quad W_{i}(t)=V_{i}(t) \cap\left[f(t, \bar{x}(t), U(t))-f^{\bar{x}}(t)\right]
$$

and

$$
\tilde{W}_{i}(t)= \begin{cases}W_{i}(t) & t \in \mathcal{A}_{i} \\ \{0\} & \text { otherwise },\end{cases}
$$

where $\mathcal{A}_{i}=\left\{t \in I: W_{i}(t) \neq \emptyset\right\}$. We claim that $V_{i}$ is measurable. Indeed, let $\mathcal{O}$ be an open subset of $X$ and call

$$
\mathcal{B}=\left\{t \in I: V_{i}(t) \cap \mathcal{O} \neq \emptyset\right\} .
$$

Since $X$ is separable, it contains a countable dense subset $\left(e_{j}\right)_{j}$. It is not difficult to check that

$$
\mathcal{B}=\bigcup_{j: e_{j} \in \mathcal{O}}\left\{t \in I:\left\langle z(t), e_{j}\right\rangle \leq-\frac{1}{i}\right\} .
$$

By (4.6), this set is measurable. Consequently, $W_{i}$ and $\tilde{W}_{i}$ are measurable. Since $\tilde{W}_{i}$ is a measurable set-valued map with closed non empty images, it has a measurable selection $v_{i}$. By $(H)(i v), v_{i}$ is integrable and satisfies

$$
v_{i}(t) \in T_{\overline{\mathrm{co}} f(t, \bar{x}(t), U(t))}\left(f^{\bar{x}}(t)\right), \quad \text { a.e. } t \in I .
$$

Hence, by (4.8) we obtain

$$
0 \leq \int_{0}^{1}\left\langle z(t), v_{i}(t)\right\rangle \mathrm{d} t \leq-\frac{1}{i} \mu\left(\mathcal{A}_{i}\right)
$$


implying that $\mu\left(\mathcal{A}_{i}\right)=0$ for any $i \in \mathbb{N}$. Therefore the set

$$
\bigcup_{i \in \mathbb{N}} \mathcal{A}_{i}=\left\{t \in I: \exists v \in\left[f(t, \bar{x}(t), U(t))-f^{\bar{x}}(t)\right] \text { satisfying }\langle z(t), v\rangle<0\right\}
$$

has measure zero. This yields the pointwise minimum principle (1.6).

Remark 4.4. As we said in section 2, the necessary conditions proved in the present section can be derived from simplified versions of the inward pointing condition, when additional regularity on the data of the problem holds. In order to obtain the same conclusions of Theorem 4.2 imposing (2.10) instead of (2.8), one may assume, for instance, that:

(a) $(\mathrm{H})$ holds, with (v) replaced by $(2.10)$, and $k_{R}, \phi \in L^{\infty}\left(I, \mathbb{R}^{+}\right)$;

(b) $F(\cdot, \bar{x})$ is continuous for any $\bar{x} \in \partial K$;

(c) $F(t, x)$ is convex for any $(t, x) \in I \times X$;

(d) $\forall R>0$ there exists a compact $\mathcal{K}_{R} \subset X$ such that $F(t, x) \subset \mathcal{K}_{R}, \forall(t, x) \in I \times R B$.

Proof. By [20, Proposition 5.2] and by the uniform continuity of the semigroup $S(\cdot)$ on compact sets, it is possible to prove that (2.10) implies

$$
\begin{aligned}
& \forall D \subset X \text { compact, } \exists \tilde{\eta}, \rho, M>0, \exists J \subset I \text { such that } \mu(J)=1 \text { and whenever } \\
& \qquad \Sigma_{\tilde{\eta}}(x ; v) \geq 0 \text { for some } t \in J, x \in D \cap \partial^{\tilde{\eta}} K, v \in F(t, x), \\
& \text { then } \exists \bar{v} \in F(t, x) \cap B(v, M) \text { satisfying } \max \left\{\Sigma_{\tilde{\eta}}(x ; \bar{v}-v) ; \Sigma_{\tilde{\eta}}(x ; \bar{v})\right\} \leq-\rho .
\end{aligned}
$$

It is not difficult to check that (2.8) can be replaced by (4.9) in the proofs of Propositions 3.2 and 3.3. On the other hand, by (d), the validity of [20, Theorem 7.1] is guaranteed by [20, Proposition 4.3]. We conclude by observing that (2.8) is not used elsewhere in the proof of Theorem 4.2 .

Remark 4.5. Analogous arguments allow to replace (2.8) by (2.11) in Theorem 4.2, when the following assumptions hold:

(a') (a), (b) and (c) of Remark 4.4, with (2.10) replaced by (2.11);

(b') $X$ is reflexive;

(c') $d_{K}$ is continuously differentiable on a neighborhood $\partial K$;

(d') either (d) of Remark 4.4 or $S(\cdot)$ is a compact semigroup.

\section{Applichtions to controlled PDEs}

Due to the general framework of our analysis, a large class of concrete models can be considered. In this section we propose as examples some optimal control problems involving wave and heat equations with different kind of boundary conditions. Various type of state constraints are allowed, including the interesting and difficult case of the pointwise state constraints, such as positivity of the solutions, so important in applications.

Example 5.1 (The Sine-Gordon equation). We consider an optimal control problem governed by the Sine-Gordon equation, used in physics to model the dynamics of a Josephson junction driven by a current source, see e.g. [27, 34]. The mathematical setting is the 
following: the unknown is the scalar function $y=y(t, \mathbf{x})$, with $\mathbf{x} \in \Omega$, a smooth and bounded domain of $\mathbb{R}^{3}, t \in I=[0,1]$, and the equation is

$$
\partial_{t t} y(t, \mathbf{x})+\partial_{t} y(t, \mathbf{x})-\Delta y(t, \mathbf{x})+\sin y(t, \mathbf{x})=u(t, \mathbf{x}) .
$$

where $u=u(t, \cdot) \in U$, a closed bounded nonempty subset of $L^{2}(\Omega)$, controls the external current driving the device. (Below we omit writing explicitly the dependence on the variable $\mathbf{x}$ ). Equation (5.1) is endowed with Dirichlet boundary conditions.

In order to handle (5.1) as system (1.1) and implement our abstract machinery to get necessary conditions, let $X=H_{0}^{1}(\Omega) \times L^{2}(\Omega)$ and

$$
x=\left(x_{1}, x_{2}\right)=\left(y, y+\partial_{t} y\right) \text {. }
$$

Then,

Now, by defining the operator

$$
\left\{\begin{array}{l}
\dot{x}_{1}(t)=x_{2}(t)-x_{1}(t) \\
\dot{x}_{2}(t)=\Delta x_{1}(t)-\sin \left(x_{1}(t)\right)+u(t) .
\end{array}\right.
$$

$$
\mathbb{A}=\left(\begin{array}{cc}
0 & I \\
\Delta & 0
\end{array}\right)
$$

with domain $D(\mathbb{A})=\left(H^{2}(\Omega) \cap H_{0}^{1}(\Omega)\right) \times H_{0}^{1}(\Omega)$, and

$$
f(t, x, u)=-\left(x_{1}, \sin \left(x_{1}\right)\right)+(0, u),
$$

(5.1) can be seen as the abstract system

$$
\dot{x}(t)=\mathbb{A} x(t)+f(t, x(t), u(t)) \quad \text { a.e. } t \in I .
$$

The operator $\mathbb{A}$ generates a strongly continuous semigroup $S(t)$ in the Hilbert space $X$. The system $\dot{x}=\mathbb{A} x$ being conservative, the state constraint $R B \subset X$, complying with the request that the energy associated with the system is bounded, satisfies the invariance assumption (2.4). Further, $f$ satisfies $(H)(i)-(i v)$, and $\partial_{x} f(t, x, u)\left(\xi_{1}, \xi_{2}\right)$ : $\mathbf{x} \mapsto-\left(\xi_{1}(\mathbf{x}), \cos \left(x_{1}(\mathbf{x})\right) \xi_{1}(\mathbf{x})\right)$ for any $\left(\xi_{1}, \xi_{2}\right) \in X$.

Since $\|S(t) x\|_{X}=\|x\|_{X}$, for any $x \in X$, and $\nabla d_{K}(x)=\frac{x}{\|x\|_{X}}$, for any $x \neq 0$, the sets $\partial^{\eta} K, \mathcal{A}^{\eta}(x)$ introduced in Section 2 take the form

$$
\partial^{\eta} K=\partial(R B)+\eta B \quad \text { and } \quad \mathcal{A}^{\eta}(x)=\cup_{\tau \in[0, \eta]}(\{\tau\} \times B(S(\tau) x, \eta)),
$$

for $x \in \partial^{\eta} K$, otherwise $\mathcal{A}^{\eta}(x)=\emptyset$. Further,

$$
\Sigma_{\eta}(x ; v)=\sup _{\tau \in[0, \eta], w \in B}\left\langle\frac{S(\tau) x+\eta w}{\|S(\tau) x+\eta w\|_{X}}, S(\tau)\left(-x_{1},-\sin \left(x_{1}\right)+v\right)\right\rangle .
$$

Using the Lipschitz regularity of the norm and taking into account Proposition 2.3, it is not difficult to prove that the inward pointing condition (2.8) for this problem can be written in the following form:

$$
\begin{gathered}
\exists \eta, \rho>0 \text { such that if, for some } x \in \partial(R B)+\eta B, v \in \overline{\mathrm{co}} U, \\
\sup _{\tau \in[0, \eta]}\left\langle S(\tau) x, S(\tau)\left(-x_{1},-\sin \left(x_{1}\right)+v\right)\right\rangle \geq-\eta\left\|\left(-x_{1},-\sin \left(x_{1}\right)+v\right)\right\|_{X}, \\
\text { then } \exists \bar{v} \in \overline{\mathrm{co}} U \text { satisfying } \\
\sup _{\tau \in[0, \eta]}\langle S(\tau) x, S(\tau)(0, \bar{v}-v)\rangle \leq-\rho .
\end{gathered}
$$


Concerning the cost functional, take a continuous function $\varphi: \mathbb{R} \rightarrow \mathbb{R}$ such that

$$
|\varphi(s)| \leq a+b|s|^{5}, \text { for some } a, b>0
$$

and define $\mathcal{G}: \mathbb{R} \rightarrow \mathbb{R}$ as

$$
\mathcal{G}(s)=\int_{0}^{s} \varphi(\sigma) d \sigma
$$

Then, $\mathcal{G}(v(\cdot)) \in L^{1}(\Omega)$, for any $v \in H_{0}^{1}(\Omega)$. The functional $G: H_{0}^{1}(\Omega) \rightarrow \mathbb{R}$ defined as

$$
G(v)=\int_{\Omega} \mathcal{G}(v(\boldsymbol{x})) \mathrm{d} \boldsymbol{x}
$$

is continuously differentiable on $H_{0}^{1}(\Omega)$ (see [1]) and

$$
\langle\nabla G(v), \xi\rangle=\int_{\Omega} \varphi(v(\mathbf{x})) \xi(\mathbf{x}) \mathrm{d} \mathbf{x}, \quad \text { for any } v, \xi \in H_{0}^{1}(\Omega) .
$$

Let $g: X \rightarrow \mathbb{R}$ be defined by $g(x)=G\left(x_{1}\right)$, for any $x=\left(x_{1}, x_{2}\right) \in H_{0}^{1}(\Omega) \times L^{2}(\Omega)$.

Now, let $(\bar{x}, \bar{u})$ be a solution of the problem of minimizing $g(x(1))$ over all admissible trajectory/control pairs $(x, u)$, such that $x(0) \in Q_{0}$, a closed subset of $X$ satisfying

$$
D_{K}(x) \cap C_{K \cap Q_{0}}(x) \neq \emptyset, \quad \forall x \in K \cap Q_{0} .
$$

From Theorem 4.2 we deduce the validity of (PMP) in normal form: for a countably additive regular measure of bounded variation $\gamma$, the solution $z=\left(z_{1}, z_{2}\right): I \rightarrow H^{-1}(\Omega) \times$ $L^{2}(\Omega)$ of the adjoint equation

$$
\left\{\begin{array}{l}
\mathrm{d} z(t)=\left(\mathbb{A}-\partial_{x} f(t, \bar{x}(t), \bar{u}(t))^{*}\right) z(t) \mathrm{d} t-\gamma(\mathrm{d} t), \quad t \in I \\
z(1)=\left(\nabla G\left(\bar{x}_{1}(1)\right), 0\right)
\end{array}\right.
$$

satisfies the minimality condition

$$
\left.\left\langle z_{2}(t), \bar{u}(t)\right)\right\rangle=\min _{u \in U}\left\langle z_{2}(t), u\right\rangle, \quad \text { for a.e. } t \in I
$$

and the transversality property

$$
-z(0) \in N_{K \cap Q_{0}}(\bar{x}(0)) .
$$

Example 5.2 (A controlled heat equation). We investigate the control of a system describing a heat transfer problem. Given $\Omega \subset \mathbb{R}^{N}$, a bounded domain with smooth boundary $\partial \Omega$, we consider the heat equation

$$
\partial_{t} x(t, \mathbf{x})-\Delta x(t, \mathbf{x})=\varphi(t, \mathbf{x})+u(t) b(\mathbf{x}) x(t, \mathbf{x}),
$$

where $\varphi \in L^{1}\left(I, L^{2}(\Omega)\right), b \in L^{\infty}(\Omega), x=x(t, \mathbf{x})$ is the temperature distribution, a function of the time $t \in I=[0,1]$ and the position $\mathbf{x} \in \Omega$, and the control $u$ takes values in the closed interval $U=[c, d]$ of $\mathbb{R}$, where $c<d$. A similar problem (without state constraints) has been studied in [3], to get second order optimality conditions. The term at the right hand side depends on a multiplicative control and represents a heat supply. We impose Dirichlet boundary conditions and define the operator $\mathbb{A}=\Delta$ with domain $D(\mathbb{A})=H^{2}(\Omega) \cap H_{0}^{1}(\Omega)$, that generates a compact strongly continuous semigroup of contractions $S(t)$ on $X=L^{2}(\Omega)$. Thus, (5.3) can be seen as the abstract system (1.1) with $f(t, x, u)=\varphi(t)+u b x$. As before, we omit the dependence on $\mathbf{x}$. It is easy to check that the hypotheses (2.5)-(2.7) are satisfied. 
Our aim is to find a temperature $x$ to be close, at the final time $t=1$, to a reference temperature $x_{D} \in X$, namely we want to minimize the functional

$$
g(x(1))=\frac{1}{2}\left\|x(1)-x_{D}\right\|_{X}^{2}
$$

among all the trajectory/control pairs $(x, u)$ satisfying the energy state constraint $K=$ $B \subset L^{2}(\Omega)$. Since $S(t)$ is a semigroup of contractions, the invariance property (2.4) holds.

Concerning the inward pointing conditions (2.8), by Remark 4.5, if $\varphi \in \mathcal{C}(I, X)$ we can consider the simplified form (2.11), namely: for some $\rho>0$ and any $t \in I$ and $x$ such that $\|x\|_{X}=1$, there exists $\bar{v} \in U$ satisfying

$$
\langle x, \varphi(t)+\bar{v} b x\rangle=\int_{\Omega} \varphi(t, \mathbf{x}) x(\mathbf{x}) d \mathbf{x}+\bar{v} \int_{\Omega} b(\mathbf{x})|x(\mathbf{x})|^{2} d \mathbf{x} \leq-\rho .
$$

Now, let $(\bar{x}, \bar{u})$ be optimal for the problem above. The assumptions of Theorem 4.2 are valid. Further, taking $Q_{0}=B\left(y_{0}, r\right) \subseteq K$, for some $y_{0} \in K$, the optimality condition holds in normal form: there exists a countably additive regular measure of bounded variation $\gamma$ such that the solution $z$ of the measure-driven adjoint variational equation

$$
\left\{\begin{array}{l}
\mathrm{d} z(t)=-(\mathbb{A}+\bar{u}(t) b) z(t) \mathrm{d} t-\gamma(\mathrm{d} t), \quad t \in I \\
z(1)=\bar{x}(1)-x_{D},
\end{array}\right.
$$

satisfies the optimality condition

$$
\bar{u}(t)\langle z(t), b \bar{x}(t)\rangle=\min _{u \in U} u\langle z(t), b \bar{x}(t)\rangle, \quad \text { for a.e. } t \in I .
$$

This condition implies that, if $\langle z(t), b \bar{x}(t)\rangle>0$, then $\bar{u}(t)=c$ and, if $\langle z(t), b \bar{x}(t)\rangle<0$, then $\bar{u}(t)=d$, a.e. in $I$.

In the last example the interesting case of pointwise constraints is discussed. This type of constraints is very useful in applications, for instance in the case of the heat equations analyzed below, imposing some pointwise bounds allows to avoid damage in the materials during the heating process. The drawback is that in our analysis we can only treat constraints $K$ having non empty interior. For this reason, suitable state spaces should be used. For instance one-dimensional Sobolev space $H^{1}(0,1)$.

Example 5.3 (A one dimensional heat equation). We analyze an optimal control problem governed by a one-dimensional heat equation modeling the heat flux in a cylindrical bar, whose length is much larger than its cross-section with perfectly insulated lateral surface: for $x=x(t, s), u(t) \in[-1,1],(t, s) \in[0,1] \times[0,1]$,

$$
\partial_{t} x(t, s)=\partial_{s s} x(t, s)-x(t, s)+u(t) \varphi(x(t, s)) .
$$

We impose Neumann boundary conditions, complying with the physical assumption that the heat flux at the two ends of the bar is zero. Let us define the operator $\tilde{\mathbb{A}}=\partial_{s s}-I$ with domain $D(\tilde{\mathbb{A}})=\left\{x \in H^{2}(0,1): x^{\prime}(0)=x^{\prime}(1)=0\right\}$ generating a strongly continuous semigroup $\tilde{S}(t)$ on $Y=L^{2}(0,1)$. The space $X=H^{1}(0,1)$ is an invariant subspace of $Y$, by the semigroups $\tilde{S}$, and the restriction of $\tilde{S}(t)$ to $X$ (called $S(t)$ ) is a strongly continuous semigroup on $X$, see [34]. Calling $\mathbb{A}$ its infinitesimal generator, we can write (5.4) in the form (1.1). Here, we assume that $f:(t, x, u) \mapsto u \varphi(x)$ satisfies $(2.5)-(2.7)$. 
The cone of nonnegative functions

$$
K=\{x \in X: x(s) \geq 0, \text { for } s \in[0,1]\} .
$$

is invariant under the action of $S(t)$. If $\forall R>0$ there exists a compact $\mathcal{K}_{R} \subset X$ such that $\varphi(x) \in \mathcal{K}_{R}, \forall x \in R B$, then by Remark 4.4 we can consider the following simplified inward pointing condition: for any $R>0$ there exists $\rho>0$ such that: for any $\bar{x} \in \partial K \cap R B$ and $v \in[-1,1]$ satisfying

$$
\sup _{\xi \in \partial d_{K}(\bar{x})} v\langle\xi, \varphi(\bar{x})\rangle_{X} \geq 0
$$

there exists $\bar{v} \in[-1,1]$ satisfying

$$
\sup _{\xi \in \partial d_{K}(\bar{x})}(\bar{v}-v)\langle\xi, \varphi(\bar{x})\rangle_{X}<-\rho .
$$

Note that this assumption is equivalent to $x \rightarrow \operatorname{sgn}\langle\xi, \varphi(x)\rangle$ is constant equal to +1 or -1 on every connected component of $\partial K \cap R B$, for all $R>0$. As remarked in [20, Section 4.3 ], in a Hilbert space we can reformulate the same condition by using normal vectors to $\partial K$. Further, for this particular set $K$, these normals belong to a compact set having a nice explicit representation (see [19]).

Let $(\bar{x}, \bar{u})$ be optimal for the problem of minimizing the functional

$$
g(x(1))=\left\|x(1)-x_{D}\right\|_{X}^{2},
$$

for a reference temperature $x_{D} \in X$, among all the admissible trajectory/control pairs $(x, u)$ satisfying the initial condition $x(0) \in K$. Then, for a measure $\gamma$ and $\lambda=1$, the solution $z$ of the adjoint equation (1.5) satisfies

$$
\bar{u}(t)\langle z(t), \varphi(\bar{x}(t))\rangle=\min _{u \in[-1,1]} u\langle z(t), \varphi(\bar{x}(t))\rangle, \quad \text { for a.e. } t \in I .
$$

\section{NECESSARY CONDITIONS With ENDPOINT CONSTRAints}

Let $Q_{1}$ be a closed subset of $X$. Consider the final constraint

$$
x(1) \in Q_{1}
$$

and the problem

$$
\text { minimize }\{g(x(1)): x \text { solution of }(1.1),(1.2),(1.3),(6.1)\} .
$$

Proposition 6.1. Assume $(H)$ and let $\bar{x}$ be locally optimal for (6.2). Then every solution of

$$
\left\{\begin{array}{l}
\dot{w}(t) \in \mathbb{A} w(t)+d \overline{c o} F\left(t, \bar{x}(t), f^{\bar{x}}(t)\right) w(t) \quad \text { a.e. } t \in I, \\
w(t) \in C_{K}(\bar{x}(t)) \quad \forall t \in I, \\
w(0) \in T_{K \cap Q_{0}}(\bar{x}(0)), w(1) \in D M_{Q_{1}}(\bar{x}(1))
\end{array}\right.
$$

satisfies

$$
\langle\nabla g(\bar{x}(1)), w(1)\rangle \geq 0
$$


Proof. Let $w$ be a solution to (6.3). Following the proof of Proposition 4.1, we can find sequences $h_{i} \rightarrow 0+$ and $w_{i} \rightarrow w(0)$ such that $\bar{x}(0)+h_{i} w_{i} \in K \cap Q_{0}$, for every $i \in \mathbb{N}$, and solutions $\bar{x}_{i}$ to

satisfying

$$
\left\{\begin{array}{l}
\dot{x}(t) \in \mathbb{A} x(t)+F(t, x(t)) \quad \text { a.e. } t \in I \\
x(t) \in K \quad \forall t \in I \\
x(0)=\bar{x}(0)+h_{i} w_{i}
\end{array}\right.
$$

$$
\frac{\bar{x}_{i}-\bar{x}}{h_{i}} \rightarrow w \text { in } \mathcal{C}(I, X)
$$

Since $w(1) \in D M_{Q_{1}}(\bar{x}(1))$, there exists $\varepsilon>0$ such that $\left.\left.\bar{x}(1)+\right] 0, \varepsilon\right](w(1)+\varepsilon B) \subset Q_{1}$. For $i$ sufficiently large, we have $h_{i} \leq \varepsilon$ and $\frac{\bar{x}_{i}(1)-\bar{x}(1)}{h_{i}} \in w(1)+\varepsilon B$, so that $\bar{x}_{i}(1)=$ $\bar{x}(1)+h_{i} \frac{\bar{x}_{i}(1)-\bar{x}(1)}{h_{i}} \in Q_{1}$. Since $\bar{x}$ is optimal for the problem (6.2), we have that

$$
g\left(\bar{x}_{i}(1)\right) \geq g(\bar{x}(1))
$$

and therefore

$$
\langle\nabla g(\bar{x}(1)), w(1)\rangle=\lim _{i \rightarrow+\infty} \frac{g\left(\bar{x}_{i}(1)\right)-g(\bar{x}(1))}{h_{i}} \geq 0
$$

Lemma 6.2. Let $C_{1} \subset X$ be a convex cone and $(x, u)$ be a trajectory/control pair satisfying (1.1). For any $t \in I$, define $\mathbb{B}(t), f^{x}(t)$ and $\mathcal{T}(t)$ as in Proposition 3.3. Assume $(H)$ (i)-(v) and that

$$
\exists \eta, \rho, M>0, \exists J \subset[1-\eta, 1] \text { such that } \mu(J)=\eta \text { and } \forall t \in J
$$

$\exists \bar{v} \in \overline{\mathrm{co}} F(t, x(t)) \cap B\left(f^{x}(t), M\right)$ satisfying $B\left(S(\tau)\left(\bar{v}-f^{x}(t)\right), 2 \rho\right) \subset C_{1}, \forall \tau<\eta$,

$$
\text { and } \Sigma_{\eta}\left(x(t) ; \bar{v}-f^{x}(t)\right) \leq-\rho \text {. }
$$

Then, for every $w_{0} \in D_{K}(x(0))$, there exists a solution $w$ of $(3.5)$ such that

$$
w(t) \in C_{K}(x(t)), \quad \forall t \in I,
$$

and $w(1) \in \operatorname{Int} C_{1}$.

The proof of Lemma 6.2 is postponed to section 7 .

Theorem 6.3. Assume $(H)$. Let $(\bar{x}, \bar{u})$ be a locally optimal pair for problem (6.2), $C_{0}$ be any convex cone contained in $T_{K \cap Q_{0}}(\bar{x}(0)), C_{1}$ be any convex cone with nonempty interior contained in $D M_{Q_{1}}(\bar{x}(1))$ and $\mathcal{S}$ be the solution operator associated with the linear evolution system

$$
\dot{w}(t)=\mathbb{A} w(t)+\partial_{x} f(t, \bar{x}(t), \bar{u}(t)) w(t) .
$$

Then, there exist $\lambda \in\{0,1\}$, a measure

$$
\gamma \in\left(\left\{w \in \mathcal{C}(I, X): w(t) \in C_{K}(\bar{x}(t)), \forall t \in I\right\}\right)^{-}
$$

and $\xi_{1} \in C_{1}^{-}$, not vanishing simultaneously, such that the function $z: I \rightarrow X^{*}$ defined by

$$
z(s)=\mathcal{S}(1, s)^{*}\left(\lambda \nabla g(\bar{x}(1))+\xi_{1}\right)+\int_{s}^{1} \mathcal{S}(t, s)^{*} \gamma(\mathrm{d} t)
$$


satisfies the minimum principle (1.6) and the transversality condition $-z(0) \in C_{0}^{-}$.

Furthermore, if $D_{K}(\bar{x}(0)) \cap C_{0} \neq \emptyset$ and $(\bar{x}, \bar{u})$ satisfies $(6.4)$, the theorem holds with $\lambda=1$.

Proof. Let $\mathcal{D}, \mathcal{E}$ be as in the proof of Theorem 4.2 and

$$
\mathcal{G}=\left\{w \in \mathcal{C}(I, X): w(1) \in C_{1}\right\}
$$

Observe that $\mathcal{D}, \mathcal{E}$ and $\mathcal{G}$ are nonempty convex cones. Furthermore, by Propositions 3.2 and 3.3 , Int $\mathcal{D} \neq \emptyset$ and since $C_{1}$ has nonempty interior, Int $\mathcal{G} \neq \emptyset$.

We have three situations:

Case 1: $\mathcal{E} \cap \mathcal{G}=\emptyset$. Then, by a separation theorem there exists $\alpha \in(\mathcal{C}(I, X))^{*}, \alpha \neq 0$, such that

$$
\inf _{e \in \mathcal{E}}\langle\alpha, e\rangle \geq \sup _{g \in \mathcal{G}}\langle\alpha, g\rangle
$$

Since $\mathcal{E}$ and $\mathcal{G}$ are cones,

$$
\alpha \in \mathcal{G}^{-} \quad \text { and } \quad-\alpha \in \mathcal{E}^{-} \text {. }
$$

Case 2: $\mathcal{E} \cap \mathcal{G} \neq \emptyset$ and $\mathcal{D} \cap(\mathcal{E} \cap \mathcal{G})=\emptyset$. Again, by a separation theorem there exists $\beta \in(\mathcal{C}(I, X))^{*}, \beta \neq 0$, such that

$$
\inf _{d \in \mathcal{D}}\langle\beta, d\rangle \geq \sup _{e \in \mathcal{E} \cap \mathcal{G}}\langle\beta, e\rangle .
$$

Since $\mathcal{D}$ and $\mathcal{E} \cap \mathcal{G}$ are cones and $\operatorname{Int} \mathcal{G} \neq \emptyset$,

$$
\beta \in(\mathcal{E} \cap \mathcal{G})^{-}=\mathcal{E}^{-}+\mathcal{G}^{-} \quad \text { and } \quad-\beta \in \mathcal{D}^{-} .
$$

Case 3, normal PMP: $\mathcal{D} \cap \mathcal{E} \cap \mathcal{G} \neq \emptyset$. Consider the linear operator $\Gamma: \mathcal{C}(I, X) \rightarrow X$ defined by $\Gamma(w)=w(1)$. We deduce from Proposition 6.1 that

$$
0 \leq\langle\nabla g(\bar{x}(1)), w(1)\rangle=\langle\nabla g(\bar{x}(1)), \Gamma w\rangle=\left\langle\Gamma^{*} \nabla g(\bar{x}(1)), w\right\rangle,
$$

for any $w \in \mathcal{D} \cap \mathcal{E} \cap \mathcal{G}$. Hence

$$
-\Gamma^{*} \nabla g(\bar{x}(1)) \in(\mathcal{D} \cap \mathcal{E} \cap \mathcal{G})^{-}=\mathcal{D}^{-}+(\mathcal{E} \cap \mathcal{G})^{-}=\mathcal{D}^{-}+\mathcal{E}^{-}+\mathcal{G}^{-} .
$$

We can put together the three cases, by stating that there exist $\beta_{1} \in \mathcal{D}^{-}, \beta_{2} \in \mathcal{E}^{-}$, $\beta_{3} \in \mathcal{G}^{-}$and $\lambda \in\{0,1\}$ satisfying

$$
-\lambda \Gamma^{*} \nabla g(\bar{x}(1))=\beta_{1}+\beta_{2}+\beta_{3} \quad \text { and } \quad\left(\lambda, \beta_{1}, \beta_{3}\right) \neq(0,0,0)
$$

(in Case 1 we set $\beta_{1}=0, \beta_{2}=-\alpha, \beta_{3}=\alpha$ and $\lambda=0$, while in Case 2 we set $\beta_{1}=-\beta$, $\beta_{2}+\beta_{3}=\beta$ and $\left.\lambda=0\right)$.

Observe that if $D_{K}(\bar{x}(0)) \cap C_{0} \neq \emptyset$ and $(\bar{x}, \bar{u})$ satisfies (6.4), then Lemma 6.2 guarantees the existence of $w \in \mathcal{D} \cap \mathcal{E} \cap \mathcal{G}$. Thus, we can consider $\lambda=1$.

Representation: Let $\gamma_{3} \in \mathcal{C}(I, X)^{*}$ be such that

$$
\left\langle\beta_{3}, y\right\rangle=\int_{0}^{1} y(t) \gamma_{3}(\mathrm{~d} t), \quad \forall y \in \mathcal{C}(I, X) .
$$


Consider $y \in \mathcal{C}(I, X)$ with $y(1)=0$. Then $y$ is in the closure of $\mathcal{G}$. Thus $\left\langle\beta_{3}, y\right\rangle \leq 0$ and $\left\langle\beta_{3},-y\right\rangle \leq 0$ implying that $\left\langle\beta_{3}, y\right\rangle=0$. Fix any $w \in \mathcal{C}(I, X)$ and define $y(\cdot) \equiv w(1)$. Then

$$
0=\left\langle\beta_{3}, w-y\right\rangle=\left\langle\beta_{3}, w\right\rangle-\int_{0}^{1} y(t) \gamma_{3}(\mathrm{~d} t)=\left\langle\beta_{3}, w\right\rangle-\left\langle\int_{0}^{1} \gamma_{3}(\mathrm{~d} t), w(1)\right\rangle .
$$

Therefore

$$
\left\langle\beta_{3}, w\right\rangle=\left\langle\int_{0}^{1} \gamma_{3}(\mathrm{~d} t), w(1)\right\rangle, \quad \forall w \in \mathcal{C}(I, X) .
$$

Set $\xi_{1}=\int_{0}^{1} \gamma_{3}(\mathrm{~d} t)$. Then for any $w \in \mathcal{G}$ we have

$$
\left\langle\xi_{1}, w(1)\right\rangle=\left\langle\beta_{3}, w\right\rangle \leq 0 .
$$

Hence $\xi_{1} \in C_{1}^{-}$.

Let us consider any solution $w$ of (4.1). We can write it in the integral form:

$$
w(t)=\mathcal{S}(t, 0) w_{0}+\int_{0}^{t} \mathcal{S}(t, s) v(s) \mathrm{d} s,
$$

where $w_{0}=w(0)$ and $v(s) \in \mathcal{T}(s)$, for a.e. $s \in I$. Then, by taking $\lambda=0$ in Case 1 and $\lambda=1$ in Case 2, and applying the characterizations of $\beta_{1} \in(C(I, X))^{*}$ and $\beta_{3} \in \mathcal{G}^{-}$, we obtain for a measure $\gamma \in \mathcal{D}^{-} \subset \mathcal{M}\left(I, X^{*}\right)$,

$$
\begin{aligned}
0 \geq & \langle-\lambda \nabla g(\bar{x}(1)), w(1)\rangle-\left\langle\beta_{1}+\beta_{3}, w\right\rangle \\
= & -\left\langle\lambda \nabla g(\bar{x}(1)), \mathcal{S}(1,0) w_{0}+\int_{0}^{1} \mathcal{S}(1, s) v(s) \mathrm{d} s\right\rangle-\left\langle\beta_{1}+\beta_{3}, w\right\rangle \\
= & -\left\langle\mathcal{S}(1,0)^{*} \lambda \nabla g(\bar{x}(1)), w_{0}\right\rangle-\int_{0}^{1}\left\langle\mathcal{S}(1, s)^{*} \lambda \nabla g(\bar{x}(1)), v(s) \mathrm{d} s\right\rangle \\
& -\int_{0}^{1}\langle w(t), \gamma(\mathrm{d} t)\rangle-\left\langle\xi_{1}, w(1)\right\rangle \\
= & -\left\langle\mathcal{S}(1,0)^{*}\left(\lambda \nabla g(\bar{x}(1))+\xi_{1}\right), w_{0}\right\rangle-\int_{0}^{1}\left\langle\mathcal{S}(1, s)^{*}\left(\lambda \nabla g(\bar{x}(1))+\xi_{1}\right), v(s) \mathrm{d} s\right\rangle \\
& -\int_{0}^{1}\left\langle\mathcal{S}(t, 0) w_{0}+\int_{0}^{t} \mathcal{S}(t, s) v(s) \mathrm{d} s, \gamma(\mathrm{d} t)\right\rangle \\
= & -\left\langle\mathcal{S}(1,0)^{*}\left(\lambda \nabla g(\bar{x}(1))+\xi_{1}\right), w_{0}\right\rangle-\int_{0}^{1}\left\langle\mathcal{S}(1, s)^{*}\left(\lambda \nabla g(\bar{x}(1))+\xi_{1}\right), v(s) \mathrm{d} s\right\rangle \\
& -\left\langle\int_{0}^{1} \mathcal{S}(t, 0)^{*} \gamma(\mathrm{d} t), w_{0}\right\rangle-\int_{0}^{1}\left\langle\int_{s}^{1} \mathcal{S}(t, s)^{*} \gamma(\mathrm{d} t), v(s)\right\rangle \mathrm{d} s .
\end{aligned}
$$

Let $z: I \rightarrow X^{*}$ be defined by (6.6). Plugging it in the inequalities above we obtain

$$
\left\langle\mathcal{S}(1,0)^{*}\left(\lambda \nabla g(\bar{x}(1))+\xi_{1}\right)+\int_{0}^{1} \mathcal{S}(t, 0)^{*} \gamma(\mathrm{d} t), w_{0}\right\rangle+\int_{0}^{1}\langle z(s), v(s) \mathrm{d} s\rangle \geq 0 .
$$

As in the proof of Theorem 4.2, taking respectively $v \equiv 0$ and $w_{0}=0$ in (6.7), we obtain the transversality condition $-z(0) \in C_{0}^{-}$and the pointwise minimum principle (1.6). 


\section{Proofs of Proposition 3.3 And Lemma 6.2}

Let $x$ be as in the statement of Proposition 3.3 and let $R:=\max _{t \in I}|x(t)|$. Consider $\eta, \rho, M, J, \phi, M_{S}$ as in (2.8), (2.7), (2.3) and define, in light of (2.6),

$$
M_{B}:=\int_{I}\|\mathbb{B}(t)\|_{L(X)} \mathrm{d} t<\infty .
$$

Let $0<\delta \leq \eta$ be such that $E \subset I, \mu(E) \leq \delta$, imply

$$
\int_{E} \phi(t) \mathrm{d} t \leq \frac{\eta}{M_{S}(1+R)}
$$

and

$$
\int_{E}\|\mathbb{B}(t)\|_{L(X)} \mathrm{d} t \leq \frac{\rho}{2 M M_{S}^{2} \mathrm{e}^{M_{S} M_{B}}} .
$$

Lemma 7.1. Let $t_{1} \in I$ and $w_{1} \in D_{K}\left(x\left(t_{1}\right)\right)$. Under all the assumptions of Proposition 3.3, there exists a solution $w$ of

$$
\left\{\begin{array}{l}
\dot{w}(t) \in \mathbb{A} w(t)+\mathbb{B}(t) w(t)+\mathcal{T}(t) \quad \text { a.e. } t \in\left[t_{1}, 1\right], \\
w\left(t_{1}\right)=w_{1},
\end{array}\right.
$$

satisfying

$$
w(t) \in D_{K}(x(t)) \quad \forall t \in\left[t_{1},\left(t_{1}+\delta\right) \wedge 1\right] .
$$

Proof. Set

$$
\begin{array}{r}
\Gamma=\left\{t \in J: \text { for some } \tau_{0} \leq \eta, S\left(\tau_{0}\right) x(t) \in \partial K+\eta B\right. \text { and } \\
\left.\sup _{z \in B\left(S\left(\tau_{0}\right) x(t), \eta\right)} \sigma\left(z ; S\left(\tau_{0}\right) f^{x}(t)\right) \geq 0\right\} .
\end{array}
$$

Then, by (2.8) and a measurable selection theorem, there exists a measurable function $\bar{v}: I \rightarrow X$ such that $\bar{v}(t) \in \overline{\mathrm{co}} f(t, x(t), U(t))$ a.e. $t \in I, \bar{v} \equiv f^{x}$ on $I \backslash \Gamma$, and, for any $t \in \Gamma$,

$$
\begin{gathered}
\left\|\bar{v}(t)-f^{x}(t)\right\|_{X} \leq M \\
\sup _{(\tau, z) \in \mathcal{A}^{\eta}(x(t))} \sigma\left(z ; S(\tau)\left(\bar{v}(t)-f^{x}(t)\right)\right) \leq-\rho .
\end{gathered}
$$

We consider the mild solution $w$ of

$$
\left\{\begin{array}{l}
\dot{w}(t)=\mathbb{A} w(t)+\mathbb{B}(t) w(t)+c_{1}\left(\bar{v}(t)-f^{x}(t)\right) \quad \text { a.e. } t \in\left[t_{1}, 1\right], \\
w\left(t_{1}\right)=w_{1},
\end{array}\right.
$$

with $c_{1} \geq 0$ to be defined below and $\bar{v}$ as in (7.5)-(7.6). Observe that $\bar{v}(t)-f^{x}(t) \in \mathcal{T}(t)$ for almost every $t \in\left[t_{1}, 1\right]$. Then, for all $t \in\left[t_{1}, 1\right]$,

$$
w(t)=S\left(t-t_{1}\right) w_{1}+\int_{t_{1}}^{t} S(t-s) \mathbb{B}(s) w(s) \mathrm{d} s+\int_{t_{1}}^{t} S(t-s) c_{1}\left(\bar{v}(s)-f^{x}(s)\right) \mathrm{d} s .
$$


Hence, by (2.3),

$$
\|w(t)\|_{X} \leq M_{S}\left\|w_{1}\right\|_{X}+\int_{t_{1}}^{t} M_{S}\|\mathbb{B}(s)\|_{L(X)}\|w(s)\|_{X} \mathrm{~d} s+\int_{t_{1}}^{t} M_{S} c_{1}\left\|\bar{v}(s)-f^{x}(s)\right\|_{X} \mathrm{~d} s .
$$

By the Gronwall lemma,

$$
\|w(t)\|_{X} \leq M_{S} \mathrm{e}^{M_{S} M_{B}}\left\|w_{1}\right\|_{X}+M_{S} \mathrm{e}^{M_{S} M_{B}} c_{1} \int_{t_{1}}^{t}\left\|\bar{v}(s)-f^{x}(s)\right\|_{X} \mathrm{~d} s .
$$

In order to complete the proof, we need to find a suitable $c_{1} \geq 0$ such that the solution $w$ of (7.7) satisfies (7.4). We consider two cases.

Case 1: $x\left(t_{1}\right) \notin \partial K$. If $x\left(\left[t_{1},\left(t_{1}+\delta\right) \wedge 1\right]\right) \cap \partial K=\emptyset$, it is clear that, for any choice of $c_{1} \geq 0$, the solution $w$ of (7.7) satisfies (7.4), because $D_{K}(x(t))=X$ for every $t \in\left[t_{1},\left(t_{1}+\delta\right) \wedge 1\right]$. Otherwise, let $\tau_{1}=\min \left\{t \in\left(t_{1},\left(t_{1}+\delta\right) \wedge 1\right]: x(t) \in \partial K\right\}$.

Observe that $\mu\left(\Gamma \cap\left[t_{1}, \tau_{1}\right]\right)>0$. Indeed, suppose by contradiction that for a.e. $t \in\left[t_{1}, \tau_{1}\right]$ and every $0 \leq \tau_{0} \leq \eta$ we have either $S\left(\tau_{0}\right) x(t) \notin \partial K+\eta B$ or

$$
\sup _{z \in B\left(S\left(\tau_{0}\right) x(t), \eta\right)} \sigma\left(z ; S\left(\tau_{0}\right) f^{x}(t)\right)<0 .
$$

By the mean value theorem (see [13]), there exists $\xi \in \partial d_{K}(z)$, for some $z$ belonging to the segment $\left[x\left(\tau_{1}\right), S\left(\tau_{1}-t_{1}\right) x\left(t_{1}\right)\right]$, satisfying

$$
d_{K}\left(x\left(\tau_{1}\right)\right)=d_{K}\left(S\left(\tau_{1}-t_{1}\right) x\left(t_{1}\right)\right)+\left\langle\xi, x\left(\tau_{1}\right)-S\left(\tau_{1}-t_{1}\right) x\left(t_{1}\right)\right\rangle .
$$

Hence, from (2.1) and (2.4) we deduce

$$
0 \leq \int_{t_{1}}^{\tau_{1}}\left\langle\xi, S\left(\tau_{1}-t\right) f^{x}(t)\right\rangle \mathrm{d} t
$$

Let $t \in\left[t_{1}, \tau_{1}\right]$. Since (2.7) and (7.2) yield

$$
\left\|x\left(\tau_{1}\right)-S\left(\tau_{1}-t\right) x(t)\right\|_{X}=\left\|\int_{t}^{\tau_{1}} S\left(\tau_{1}-s\right) f^{x}(s) \mathrm{d} s\right\|_{X} \leq M_{S}(1+R) \int_{t}^{\tau_{1}} \phi(s) \mathrm{d} s \leq \eta,
$$

we know that $S\left(\tau_{1}-t\right) x(t) \in \partial K+\eta B$. Moreover,

$$
\left\|z-S\left(\tau_{1}-t\right) x(t)\right\|_{X} \leq\left\|x\left(\tau_{1}\right)-S\left(\tau_{1}-t\right) x(t)\right\|_{X} \leq \eta .
$$

Therefore, setting $\tau_{0}=\tau_{1}-t$ and combining (7.10) and (7.11), we obtain a contradiction. Define

$$
c_{1}= \begin{cases}\frac{4\left\|w_{1}\right\|_{X}\left(M_{S}+M_{S}^{2} M_{B} \mathrm{e}^{M_{S} M_{B}}\right)}{\rho \mu\left(\Gamma \cap\left[t_{1}, \tau_{1}\right]\right)} & \text { if } w_{1} \neq 0 \\ 1 & \text { otherwise } .\end{cases}
$$

For every $t \in\left[t_{1}, \tau_{1}\right)$, we have $w(t) \in D_{K}(x(t))=X$. Let $t \in\left[\tau_{1},\left(t_{1}+\delta\right) \wedge 1\right]$. Again, if $x(t) \notin \partial K$, then $w(t) \in D_{K}(x(t))=X$. Otherwise, $x(t) \in \partial K$ implies, in view of (7.2), that $(t-s, x(t)) \in \mathcal{A}^{\eta}(x(s))$ for every $s \in\left[t_{1}, t\right]$. Consequently, by (7.3), (7.6), (7.8), (7.9) 
and the definition of $c_{1}$, for any $\xi \in \partial d_{K}(x(t))$ we obtain

$$
\begin{aligned}
\langle\xi, w(t)\rangle= & \left\langle\xi, S\left(t-t_{1}\right) w_{1}\right\rangle+\int_{t_{1}}^{t}\langle\xi, S(t-s) \mathbb{B}(s) w(s)\rangle \mathrm{d} s \\
& +\int_{t_{1}}^{t}\left\langle\xi, S(t-s) c_{1}\left(\bar{v}(s)-f^{x}(s)\right)\right\rangle \mathrm{d} s \\
\leq & M_{S}\left\|w_{1}\right\|_{X}+\int_{t_{1}}^{t} M_{S}\|\mathbb{B}(s)\|_{L(X)}\left(M_{S} \mathrm{e}^{M_{S} M_{B}}\left\|w_{1}\right\|_{X}\right. \\
& \left.+M_{S} \mathrm{e}^{M_{S} M_{B}} c_{1} \int_{t_{1}}^{s}\left\|\bar{v}(r)-f^{x}(r)\right\|_{X} \mathrm{~d} r\right) \mathrm{d} s-\rho c_{1} \mu\left(\Gamma \cap\left[t_{1}, t\right]\right) \\
\leq & \left\|w_{1}\right\|_{X}\left(M_{S}+M_{S}^{2} M_{B} \mathrm{e}^{M_{S} M_{B}}\right) \\
& -c_{1} \mu\left(\Gamma \cap\left[t_{1}, t\right]\right)\left(\rho-M M_{S}^{2} \mathrm{e}^{M_{S} M_{B}} \int_{t_{1}}^{t}\|\mathbb{B}(s)\|_{L(X)} \mathrm{d} s\right) \\
\leq & \left\|w_{1}\right\|_{X}\left(M_{S}+M_{S}^{2} M_{B} \mathrm{e}^{M_{S} M_{B}}\right)-c_{1} \mu\left(\Gamma \cap\left[\tau_{1}, t\right]\right) \frac{\rho}{2}<0 .
\end{aligned}
$$

Since $\partial d_{K}(x(t))$ is weakly*-compact, we can conclude that $w(t) \in D_{K}(x(t))$.

Case 2: $x\left(t_{1}\right) \in \partial K$. Since $w_{1} \in D_{K}\left(x\left(t_{1}\right)\right)$, we can find $\tau_{0} \in\left(t_{1},\left(t_{1}+\delta\right) \wedge 1\right]$ such that

$$
\sigma\left(x(t) ; S\left(t-t_{1}\right) w_{1}\right)+M_{S}^{2} \mathrm{e}^{M_{S} M_{B}}\left\|w_{1}\right\|_{X} \int_{t_{1}}^{t}\|\mathbb{B}(s)\|_{L(X)} \mathrm{d} s<0, \quad \forall t \in\left(t_{1}, \tau_{0}\right] .
$$

Indeed, assume by contradiction that there exist sequences $t^{i} \rightarrow t_{1}+$ and $\xi_{i} \in \partial d_{K}\left(x\left(t^{i}\right)\right)$ satisfying

$$
\left\langle\xi_{i}, S\left(t^{i}-t_{1}\right) w_{1}\right\rangle \geq-\frac{1}{i}, \quad \forall i \in I
$$

Assuming (up to a subsequence) that

$$
\xi_{i} \stackrel{*}{\rightarrow} \xi \text { weakly-star in } X^{*},
$$

for some $\xi \in \partial d_{K}\left(x\left(t_{1}\right)\right)$ (here we use the weak ${ }^{\star}$-upper semicontinuity of Clarke gradient, see $[13])$, and passing to the limit in (7.13), we obtain the contradictory

$$
\left\langle\xi, w_{1}\right\rangle \geq 0
$$

If $x\left(\left(t_{1}, \tau_{0}\right]\right) \cap \partial K=\emptyset$, then for any choice of $c_{1} \geq 0$, we have $w(t) \in D_{K}(x(t))=X$ for all $t \in\left(t_{1}, \tau_{0}\right]$, and we can repeat the same reasoning as in Case 1 (replacing $t_{1}$ by $\tau_{0}$ ) in order to define a suitable $c_{1}$ such that the associated solution $w$ of (7.7) satisfies (7.4).

Otherwise, let $\tau_{1} \in\left(t_{1}, \tau_{0}\right]$ be such that $x\left(\tau_{1}\right) \in \partial K$. As in Case 1 , we can prove that

$$
\mu\left(\Gamma \cap\left(t_{1}, t\right]\right)>0 \quad \forall t \in\left(t_{1}, \tau_{0}\right] \text { such that } x(t) \in \partial K .
$$

In particular, we can define

$$
c_{1}=\frac{4\left\|w_{1}\right\|_{X}\left(M_{S}+M_{S}^{2} M_{B} \mathrm{e}^{M_{S} M_{B}}\right)}{\rho \mu\left(\Gamma \cap\left[t_{1}, \tau_{1}\right]\right)} .
$$


Let $t \in\left(t_{1}, \tau_{1}\right]$. If $x(t) \notin \partial K$, then $w(t) \in D_{K}(x(t))=X$. In the case $x(t) \in \partial K$, by (7.3), (7.8), (7.9), (7.12), (7.14) and the definition of $\tau_{0}$, we obtain for any $\xi \in \partial d_{K}(x(t))$,

$$
\begin{aligned}
& \langle\xi, w(t)\rangle=\left\langle\xi, S\left(t-t_{1}\right) w_{1}\right\rangle+\int_{t_{1}}^{t}\langle\xi, S(t-s) \mathbb{B}(s) w(s)\rangle \mathrm{d} s \\
& +\int_{t_{1}}^{t}\left\langle\xi, S(t-s) c_{1}\left(\bar{v}(s)-f^{x}(s)\right)\right\rangle \mathrm{d} s \\
& \leq\left\langle\xi, S\left(t-t_{1}\right) w_{1}\right\rangle+\int_{t_{1}}^{t} M_{S}\|\mathbb{B}(s)\|_{L(X)}\|w(s)\|_{X} \mathrm{~d} s-\rho c_{1} \mu\left(\Gamma \cap\left[t_{1}, t\right]\right) \\
& \leq\left\langle\xi, S\left(t-t_{1}\right) w_{1}\right\rangle+M_{S}^{2} \mathrm{e}^{M_{S} M_{B}}\left\|w_{1}\right\|_{X} \int_{t_{1}}^{t}\|\mathbb{B}(s)\|_{L(X)} \mathrm{d} s \\
& +M_{S}^{2} \mathrm{e}^{M_{S} M_{B}} \int_{t_{1}}^{t}\|\mathbb{B}(s)\|_{L(X)}\left(\int_{t_{1}}^{s} c_{1}\left\|\bar{v}(r)-f^{x}(r)\right\|_{X} \mathrm{~d} r\right) \mathrm{d} s-\rho c_{1} \mu\left(\Gamma \cap\left[t_{1}, t\right]\right) \\
& <c_{1} \mu\left(\Gamma \cap\left[t_{1}, t\right]\right)\left(M_{S}^{2} \mathrm{e}^{M_{S} M_{B}} M \int_{t_{1}}^{t}\|\mathbb{B}(s)\|_{L(X)} \mathrm{d} s-\rho\right)<0 .
\end{aligned}
$$

Now, consider $t \in\left[\tau_{1},\left(t_{1}+\delta\right) \wedge 1\right]$. It is enough to consider the case $x(t) \in \partial K$, the other one being trivial. Since $\mu\left(\Gamma \cap\left[t_{1}, t\right]\right) \geq \mu\left(\Gamma \cap\left[t_{1}, \tau_{1}\right]\right)>0$, arguing as above and applying (7.3), (7.8), (7.9), (7.15), we obtain that for any $\xi \in \partial d_{K}(x(t))$

$$
\begin{aligned}
\langle\xi, w(t)\rangle= & \left\langle\xi, S\left(t-t_{1}\right) w_{1}\right\rangle+\int_{t_{1}}^{t}\langle\xi, S(t-s) \mathbb{B}(s) w(s)\rangle \mathrm{d} s \\
& +\int_{t_{1}}^{t}\left\langle\xi, S(t-s) c_{1}\left(\bar{v}(s)-f^{x}(s)\right)\right\rangle \mathrm{d} s \\
\leq & M_{S}\left\|w_{1}\right\|_{X}+\int_{t_{1}}^{t} M_{S}\|\mathbb{B}(s)\|_{L(X)}\left(M_{S} \mathrm{e}^{M_{S} M_{B}}\left\|w_{1}\right\|_{X}\right. \\
& \left.\quad+M_{S} \mathrm{e}^{M_{S} M_{B}} c_{1} \int_{t_{1}}^{s}\left\|\bar{v}(r)-f^{x}(r)\right\|_{X} \mathrm{~d} r\right)-\rho c_{1} \mu\left(\Gamma \cap\left[t_{1}, t\right]\right) \\
\leq & \left\|w_{1}\right\|_{X}\left(M_{S}+M_{S}^{2} M_{B} \mathrm{e}^{M_{S} M_{B}}\right) \\
& -c_{1} \mu\left(\Gamma \cap\left[t_{1}, t\right]\right)\left(\rho-M M_{S}^{2} \mathrm{e}^{M_{S} M_{B}} \int_{t_{1}}^{t}\|\mathbb{B}(s)\|_{L(X)} \mathrm{d} s\right)<0,
\end{aligned}
$$

completing the proof.

Proof of Proposition 3.3. Let $(x, u)$ be an admissible pair for (1.1), (1.3) and let $w_{0} \in$ $D_{K}(x(0))$. By Lemma 7.1 we can construct by iteration a solution $w$ of (3.5) that satisfies

$$
w(t) \in D_{K}(x(t)), \quad \forall t \in I .
$$

It remains to prove that $w$ belongs to the interior of (3.6). Consider the compact set

$$
\mathcal{A}=\{t \in I: x(t) \in \partial K\} .
$$


Notice that, if $t \notin \mathcal{A}$, then for any $\varepsilon>0$ we have $w(t)+\varepsilon B \subset D_{K}(x(t))=X$. We claim that there exists $\varepsilon>0$ such that

$$
\sigma(x(t) ; w(t)) \leq-2 \varepsilon, \quad \forall t \in \mathcal{A} .
$$

Indeed, otherwise, we can find sequences $t_{i} \in \mathcal{A}$ and $\xi_{i} \in \partial d_{K}\left(\bar{x}\left(t_{i}\right)\right)$ such that

$$
\left\langle\xi_{i}, w\left(t_{i}\right)\right\rangle>-\frac{1}{i}
$$

Then, taking subsequences and keeping the same notations,

$$
t_{i} \rightarrow t \in \mathcal{A}, \quad \xi_{i} \stackrel{*}{\rightarrow} \xi \text { weakly-star in } X^{*},
$$

for some $\xi \in \partial d_{K}(x(t))$, leading to the contradictory

$$
\langle\xi, w(t)\rangle \geq 0 .
$$

Hence, for any $t \in \mathcal{A}$, any $\xi \in \partial d_{K}(x(t))$ and any $z \in B$, we have

$$
\langle\xi, w(t)+\varepsilon z\rangle \leq\langle\xi, w(t)\rangle+\varepsilon \leq-\varepsilon
$$

Finally, recalling Lemma 3.1, we obtain

$$
w(t)+\varepsilon B \subset D_{K}(x(t)) \subset C_{K}(x(t)), \quad \forall t \in I .
$$

Proof of Lemma 6.2. Let $R:=\max _{t \in I}|x(t)|$ and consider $\eta, \rho, M, M_{S}$ as in (6.4), (2.3) and $M_{B}$ as in (7.1). Let $0<\delta \leq \eta$ be such that

$$
\int_{1-\delta}^{1}\|\mathbb{B}(t)\|_{L(X)} \mathrm{d} t<\frac{\rho}{2 M M_{S}^{2} \mathrm{e}^{M_{S} M_{B}}} \quad \text { and } \quad \int_{1-\delta}^{1} \phi(t) \mathrm{d} t \leq \frac{\eta}{M_{S}(1+R)} .
$$

Given $w_{0} \in D_{K}(x(0))$, by Lemma 7.1 we can construct by iteration a solution $w:[0,1-$ $\delta] \rightarrow X$ of (3.5) that satisfies

$$
w(t) \in D_{K}(x(t)), \quad \forall t \in[0,1-\delta] .
$$

Call $w_{1}=w(1-\delta)$. Since $w_{1} \in D_{K}(x(1-\delta))$, as in the proof of Lemma 7.1 we can find $\tau_{0} \in(1-\delta, 1]$ such that

$(7.17)$

$$
\sigma\left(x(t) ; S(t-(1-\delta)) w_{1}\right)+M_{S}^{2} \mathrm{e}^{M_{S} M_{B}}\left\|w_{1}\right\|_{X} \int_{1-\delta}^{t}\|\mathbb{B}(s)\|_{L(X)} \mathrm{d} s<0, \quad \forall t \in\left(1-\delta, \tau_{0}\right] .
$$

By (6.4) and a measurable selection theorem, there exists a measurable function $\bar{v}$ : $[1-\delta, 1] \rightarrow X$ such that for almost every $t \in[1-\delta, 1], \bar{v}(t) \in \overline{\mathrm{co}} f(t, x(t), U(t))$,

$$
\begin{aligned}
\left\|\bar{v}(t)-f^{x}(t)\right\|_{X} & \leq M, \\
B\left(S(\tau)\left(\bar{v}(t)-f^{x}(t)\right), 2 \rho\right) & \subset C_{1}, \quad \forall \tau<\eta,
\end{aligned}
$$

and

$$
\Sigma_{\eta}\left(x(t) ; \bar{v}-f^{x}(t)\right) \leq-\rho .
$$

Extend $w$ on $I$ by considering on $[1-\delta, 1]$ the mild solution of

$$
\left\{\begin{array}{l}
\dot{w}(t)=\mathbb{A} w(t)+\mathbb{B}(t) w(t)+c\left(\bar{v}(t)-f^{x}(t)\right) \quad \text { a.e. } t \in[1-\delta, 1] \\
w(1-\delta)=w_{1}
\end{array}\right.
$$


where $c>0$ satisfies

$$
c>\frac{2 M_{S}\left\|w_{1}\right\|_{X}\left(1+M_{S} M_{B} \mathrm{e}^{M_{S} M_{B}}\right)}{\left(\tau_{0}-(1-\delta)\right) \rho} .
$$

Observe that $w$ is a solution of $(3.5)$ on $I$ and that for every $t \in[1-\delta, 1]$ we have

$$
w(t)=S(t+\delta-1) w_{1}+\int_{1-\delta}^{t} S(t-s) \mathbb{B}(s) w(s) \mathrm{d} s+\int_{1-\delta}^{t} S(t-s) c\left(\bar{v}(s)-f^{x}(s)\right) \mathrm{d} s .
$$

We can deduce, applying (2.3), that

$$
\|w(t)\|_{X} \leq M_{S}\left\|w_{1}\right\|_{X}+\int_{1-\delta}^{t} M_{S}\|\mathbb{B}(s)\|_{L(x)}\|w(s)\|_{X} \mathrm{~d} s+\int_{1-\delta}^{t} M_{S} c\left\|\bar{v}(s)-f^{x}(s)\right\|_{X} \mathrm{~d} s .
$$

By the Gronwall lemma we finally obtain the estimate

$$
\|w(t)\|_{X} \leq M_{S} \mathrm{e}^{M_{S} M_{B}}\left\|w_{1}\right\|_{X}+M_{S} \mathrm{e}^{M_{S} M_{B}} c \int_{1-\delta}^{t}\left\|\bar{v}(s)-f^{x}(s)\right\|_{X} \mathrm{~d} s, \quad \forall t \in[1-\delta, 1] .
$$

By (7.19) and the convexity of $C_{1}$, we obtain

$$
B\left(\int_{1-\delta}^{1} S(1-s) c\left(\bar{v}(s)-f^{x}(s)\right) \mathrm{d} s, 2 \delta \rho c\right) \subset \overline{C_{1}} .
$$

Moreover, by (7.16), (7.18), (7.21) and (7.22), we have

$$
\begin{aligned}
\| S(\delta) w_{1} & +\int_{1-\delta}^{1} S(1-s) \mathbb{B}(s) w(s) \mathrm{d} s\left\|_{X} \leq M_{S}\right\| w_{1}\left\|_{X}+\int_{1-\delta}^{1} M_{S}\right\| \mathbb{B}(s)\left\|_{L(X)}\right\| w(s) \|_{X} \mathrm{~d} s \\
& \leq M_{S}\left\|w_{1}\right\|_{X}+M_{S} \int_{1-\delta}^{1}\|\mathbb{B}(s)\|_{L(X)} \mathrm{d} s\left(M_{S} \mathrm{e}^{M_{S} M_{B}}\left\|w_{1}\right\|_{X}+M_{S} \mathrm{e}^{M_{S} M_{B}} c \delta M\right) \\
& \leq M_{S}\left\|w_{1}\right\|_{X}\left(1+M_{S} M_{B} \mathrm{e}^{M_{S} M_{B}}\right)+\delta M M_{S}^{2} \mathrm{e}^{M_{S} M_{B}} c \int_{1-\delta}^{1}\|\mathbb{B}(s)\|_{L(X)} \mathrm{d} s \leq \delta \rho c .
\end{aligned}
$$

Therefore,

$$
\begin{aligned}
w(1) & =S(\delta) w_{1}+\int_{1-\delta}^{1} S(1-s) B(s) w(s) \mathrm{d} s+\int_{1-\delta}^{1} S(1-s) c\left(\bar{v}(s)-f^{x}(s)\right) \mathrm{d} s \\
& \in B\left(\int_{1-\delta}^{1} S(1-s) c\left(\bar{v}(s)-f^{x}(s)\right) \mathrm{d} s, \delta \rho c\right) \subset \operatorname{Int} C_{1} .
\end{aligned}
$$

It remains to verify (6.5). If $t \in[0,1-\delta]$, we have $w(t) \in D_{K}(x(t)) \subseteq C_{K}(x(t))$ by Proposition 3.1. Let us prove that $w(t) \in D_{K}(x(t))$ also for $t \in(1-\delta, 1]$.

Let $t \in\left(1-\delta, \tau_{0}\right]$ be such that $x(t) \in \partial K$, otherwise $w(t) \in D_{K}(x(t))=X$ and the claim is trivial. Observe that by (7.16) for every $s \in[1-\delta, t]$ we have

$$
\|x(t)-S(t-s) x(s)\|_{X}=\left\|\int_{s}^{t} S(t-r) f^{x}(r) \mathrm{d} r\right\|_{X} \leq M_{S}(1+R) \int_{s}^{t} \phi(r) \mathrm{d} r \leq \eta .
$$


In particular, $S(t-s) x(s) \in \partial K+\eta B$. Therefore, by (7.16), (7.17), (7.18), (7.20) and (7.22), we obtain for any $\xi \in \partial d_{K}(x(t))$,

$$
\begin{aligned}
& \langle\xi, w(t)\rangle=\left\langle\xi, S(t-(1-\delta)) w_{1}\right\rangle+\int_{1-\delta}^{t}\langle\xi, S(t-s) \mathbb{B}(s) w(s)\rangle \mathrm{d} s \\
& +\int_{1-\delta}^{t}\left\langle\xi, S(t-s) c\left(\bar{v}(s)-f^{x}(s)\right)\right\rangle \mathrm{d} s \\
& \leq\left\langle\xi, S(t-(1-\delta)) w_{1}\right\rangle+\int_{1-\delta}^{t} M_{S}\|\mathbb{B}(s)\|_{L(X)}\|w(s)\|_{X} \mathrm{~d} s-\rho c(t-(1-\delta)) \\
& \leq\left\langle\xi, S(t-(1-\delta)) w_{1}\right\rangle+M_{S}^{2} \mathrm{e}^{M_{S} M_{B}}\left\|w_{1}\right\|_{X} \int_{1-\delta}^{t}\|\mathbb{B}(s)\|_{L(X)} \mathrm{d} s \\
& +M_{S}^{2} \mathrm{e}^{M_{S} M_{B}} \int_{1-\delta}^{t}\|\mathbb{B}(s)\|_{L(X)}\left(\int_{1-\delta}^{s} c\left\|\bar{v}(r)-f^{x}(r)\right\|_{X} \mathrm{~d} r\right) \mathrm{d} s-\rho c(t-(1-\delta)) \\
& <c(t-(1-\delta))\left(M_{S}^{2} \mathrm{e}^{M_{S} M_{B}} M \int_{1-\delta}^{1}\|\mathbb{B}(s)\|_{L(X)} \mathrm{d} s-\rho\right)<0 .
\end{aligned}
$$

Now, consider $t \in\left(\tau_{0}, 1\right]$ such that $x(t) \in \partial K$. Arguing as above and applying (7.16), (7.18), (7.20), (7.21) and (7.22), we obtain that for any $\xi \in \partial d_{K}(x(t))$

$$
\begin{aligned}
\langle\xi, w(t)\rangle=\left\langle\xi, S(t-(1-\delta)) w_{1}\right\rangle+\int_{1-\delta}^{t}\langle\xi, S(t-s) \mathbb{B}(s) w(s)\rangle \mathrm{d} s & \\
& \quad+\int_{1-\delta}^{t}\left\langle\xi, S(t-s) c\left(\bar{v}(s)-f^{x}(s)\right)\right\rangle \mathrm{d} s \\
\leq & M_{S}\left\|w_{1}\right\|_{X}+\int_{1-\delta}^{t} M_{S}\|\mathbb{B}(s)\|_{L(X)}\left(M_{S} \mathrm{e}^{M_{S} M_{B}}\left\|w_{1}\right\|_{X}\right. \\
& \left.\quad+M_{S} \mathrm{e}^{M_{S} M_{B}} c \int_{1-\delta}^{s}\left\|\bar{v}(r)-f^{x}(r)\right\|_{X} \mathrm{~d} r\right)-\rho c(t-(1-\delta)) \\
\leq & M_{S}\left\|w_{1}\right\|_{X}\left(1+M_{S} M_{B} \mathrm{e}^{M_{S} M_{B}}\right) \\
& \quad-c(t-(1-\delta))\left(\rho-M M_{S}^{2} \mathrm{e}^{M_{S} M_{B}} \int_{1-\delta}^{t}\|\mathbb{B}(s)\|_{L(X)} \mathrm{d} s\right)<0,
\end{aligned}
$$

completing the proof.

Acknowledgments. We would like to thank Professor Vittorino Pata for valuable suggestions.

\section{REFERENCES}

[1] A. Ambrosetti, G. Prodi, A primer of nonlinear analysis. Cambridge Studies in Advanced Mathematics, 34, Cambridge University Press, Cambridge, 1993.

[2] N. Arada, J.-P. Raymond, Dirichlet boundary control of semilinear parabolic equations part 2: problems with pointwise state constraints, Appl. Math. Optim.45 (2002), 145-167.

[3] M.S. Aronna, J.F. Bonnans, A. Kröner, Optimal control of infinite dimensional bilinear systems: application to the heat and wave equation, Math. Program., Ser. B, Springer, 2016, pp.32.

[4] J.-P. Aubin, H. Frankowska, Set-Valued Analysis, Birkhäuser, Boston, Basel, Berlin, 1990. 
[5] V. Barbu, Constrained control problems with convex cost in Hilbert space, J. Math. Anal. Appl. 56 (1976), 502-528.

[6] V. Barbu, Analysis and control of nonlinear infinite dimensional systems, Academic Press, Boston, New York, 1993.

[7] V. Barbu, G. Wang, State constraint optimal control problems governed by semilinear equations, Numer. Funct. Anal. Optim. 21 (2000), 411-424.

[8] A. Bensoussan, G. Da Prato, M. Delfour, S. K. Mitter, Representation and control of infinite dimensional systems, vol.1, Birkhäuser, Boston, 1992.

[9] A. Bensoussan, G. Da Prato, M. Delfour, S. K. Mitter, Representation and control of infinite dimensional systems, vol.2, Birkhäuser, Boston, 1993.

[10] J.F. Bonnans, P. Jaisson, Optimal control of a parabolic equation with time-dependent state constraints, SIAM J. Control Optim. 48 (2010), 4550-4571.

[11] E. Casas, Pontryagin's principle for state-constrained boundary control problems of semilinear parabolic equations, SIAM J. Control Optim. 35 (), 1297-1327.

[12] A. Cernea, H. Frankowska, A connection between the maximum principle and dynamic programming for constrained control problems, SIAM J. Control Optim. 4 (2005), 673-703.

[13] F. H. Clarke, Optimization and nonsmooth analysis, SIAM, Philadelphia, 1990.

[14] Yu. B. Egorov, Optimal control in Banach spaces, Dokl. Akad. Nauk SSSR 150 (1962), 241-244.

[15] Yu. B. Egorov, Some necessary conditions for optimality in Banach spaces, Mat. Sbornik 64 (1964), $79-101$.

[16] H. O. Fattorini, Optimal control problems with state constraints for semilinear distributed-parameter system, J. Optim. Theory Appl. 88 (1996), 25-59.

[17] H. O. Fattorini, Infinite-dimensional optimization and control theory, Cambridge University Press, Cambridge, 1999.

[18] H. Frankowska, A priori estimates for operational differential inclusions, J. Differential Equations 84 (1990), 100-128.

[19] H. Frankowska, E.M. Marchini, M. Mazzola, A relaxation result for state constrained inclusions in infinite dimension, Math. Control Relat. Fields 6 (2016), 113-141.

[20] H. Frankowska, E.M. Marchini, M. Mazzola, Distance estimates for state constrained trajectories of infinite dimensional differential inclusions, ESAIM: COCV2017, doi: https://doi.org/10.1051/cocv/2017032

[21] H. Frankowska, D. Tonon, Inward pointing trajectories, normality of the maximum principle and the non occurrence of the Lavrentieff phenomenon in optimal control under state constraints, J. Convex Anal. 20 (2013), 1147-1180.

[22] H. Frankowska, H. Zhang, X. Zhang, End points constrained stochastic optimal control problems, SIAM J. Control Optim., to appear.

[23] B. Hu, J. Yong, Pontryagin maximum principle for semilinear and quasi-linear parabolic equations with pointwise state constraints, SIAM J. Control Optim. 33 (1995), 1857-1880.

[24] I. Lasiecka, R. Triggiani, Control theory for partial differential equations: continuous and approximation theories, Cambridge University Press, Cambridge, 2000.

[25] X. Li, J. Yong, Optimal control theory for infinite-dimensional systems, Birkhäuser, Boston, 1995.

[26] H. Liu, J. Yang, Optimal control of semilinear parabolic systems with state constraint, J. Math. Anal. Appl. 417 (2014), 787-803.

[27] P.S. Lomdhal, O.H. Soerensen, P.L. Christiansen, Soliton excitations in Josephson tunnel junctions, Phys. Rev. B 25 (1982), 5737-5748.

[28] I. Neitzel, F. Tröltzsch, On regularization methods for the numerical solution of parabolic control problems with pointwise state constraints, ESAIM Control Optim. Calc. Var. 15 (2009), 426-453.

[29] A. Pazy, Semigroups of linear operators and applications to partial differential equations, SpringerVerlag, New York, 1983.

[30] P. Raymond, H. Zidani, Pontryagins principle for state-constrained control problems governed by parabolic equations with unbounded controls, SIAM J. Control Optim. 36 (1998), 1853-1879. 
[31] F. Rampazzo, R.B. Vinter, A theorem on existence of neighbouring trajectories satisfying a state constraint, with applications to optimal control, IMA J. Math. 16 (1999), 335-351.

[32] F. Rampazzo, R.B. Vinter, Degenerate optimal control problems with state constraints, SIAM J. Control Optim. 39 (2000), 989-1007.

[33] H. M. Soner, Optimal control with state-space constraints, SIAM J. Control Optim. 24 (1986), $552-$ 561.

[34] R. Temam, Infinite-dimensional dynamical systems in mechanics and physics, Springer, New York, 1997.

[35] F. Tröltzsch, Optimal control of partial differential equations: theory, methods and applications, AMS, Providence, 2010.

CNRS, Institut de Mathématiques de Jussieu - Paris Rive Gauche, Université Sorbonne, Case 247, 4 Place Jussieu, 75252 Paris, France

E-mail address: helene.frankowska@imj-prg.fr

Dipartimento di Matematica "F.Brioschi", Politecnico di Milano

Piazza Leonardo da Vinci 32, 20133 Milano, Italy

E-mail address: elsa.marchini@polimi.it

Université Sorbonne, Institut de Mathématiques de Jussieu - Paris Rive Gauche, CNRS, Case 247, 4 Place Jussieu, 75252 Paris, France

E-mail address: marco.mazzola@imj-prg.fr 